\title{
MicroRNA-451: epithelial-mesenchymal transition inhibitor and prognostic biomarker of hepatocelluar carcinoma
}

\author{
Jia-Yuan Huang ${ }^{1, *}$, Kai Zhang ${ }^{1, *}$, Dong-Qin Chen ${ }^{1, *}$, Jing Chen ${ }^{1}$, Bing Feng ${ }^{1}$, Haizhu \\ Song ${ }^{1}$, Yitian Chen ${ }^{1}$, Ziman Zhu ${ }^{2}$, Lei Lu ${ }^{3}$, Wei De ${ }^{4}$, Rui Wang ${ }^{1}$ and Long-Bang Chen ${ }^{1}$ \\ ${ }^{1}$ Department of Medical Oncology, Jinling Hospital, School of Medicine, Nanjing University, Nanjing, Jiangsu, China \\ ${ }^{2}$ Department of Hepatobiliary Surgery, First Hospital Affiliated to the Chinese PLA General Hospital, Beijing, China \\ ${ }^{3}$ Liver Disease Center of PLA, The 81th Hospital of PLA, Nanjing, Jiangsu, China \\ ${ }^{4}$ Department of Biochemistry and Molecular Biology, Nanjing Medical University, Nanjing, Jiangsu, China \\ * These authors have contributed equally to this work \\ Correspondence to: Rui Wang, email: wangrui218@163.com
}

Long-Bang Chen, email: chenlongbang@yeah.net

Keywords: hepatocellular carcinoma, miR-451, c-Myc, epithelial-mesenchymal transition, invasion

Received: December 12,2014 Accepted: May 12, $2015 \quad$ Published: May 27, 2015

This is an open-access article distributed under the terms of the Creative Commons Attribution License, which permits unrestricted use, distribution, and reproduction in any medium, provided the original author and source are credited.

\section{ABSTRACT}

Increasing evidence indicates that dysregulation of microRNAs (miRNAs) plays critical roles in malignant transformation and tumor progression. Previously, we have shown that microRNA-451 (miR-451) inhibits growth, increases chemo- or radiosensitivity and reverses epithelial to mesenchymal transition (EMT) in lung cancer. However, the roles of miR-451 in hepatocelluar carcinoma (HCC) progression and metastasis are still largely unknown. Reduced miR-451 in HCC tissues was observed to be significantly correlated with advanced clinical stage, metastasis and worse disease-free or overall survival. Through gain- and loss-of function experiments, we demonstrated that miR-451 inhibited cell growth, induced G0/G1 arrest and promoted apoptosis in HCC cells. Importantly, miR-451 could inhibit the migration and invasion in vitro, as well as in vivo metastasis of HCC cells through regulating EMT process. Moreover, the oncogene c-Myc was identified as a direct and functional target of miR-451 in HCC cells. Knockdown of c-Myc phenocopied the effects of miR-451 on EMT and metastasis of HCC cells, whereas overexpression of c-Myc partially attenuated the functions of miR-451 restoration. Furthermore, miR451 downregulation-induced c-Myc overexpression leads to the activation of Erk1/2 signaling, which induces acquisition of EMT phenotype through regulation of GSK-3 $\beta$ / snail/E-cadherin and the increased expression of MMPs family members in HCC cells. Collectively, these data demonstrated that miR-451 is a novel prognostic biomarker for HCC patients and that function as a potential metastasis inhibitor in HCC cells through activation of the Erk1/2 signaling, at least partially by targeting c-Myc. Thus, targeting miR-451/c-Myc/Erk1/2 axis may be a potential strategy for the treatment of metastatic HCC.

\section{INTRODUCTION}

Hepatocellular carcinoma (HCC) is the fifth common cause of cancer related death worldwide [1]. Although various therapies are used to improve outcomes of HCC patients, such as hepatic resection or liver transplantation, the 5-year survival rate remains only $30 \%$ [2]. A major of HCC patients usually cannot have curative surgery which is largely because of distant metastasis and high recurrence ratio at the time of diagnosis [3]. Therefore, a better understanding the molecular mechanisms involved in hepatocellular carcinogenesis contributes to identification 
Table 1: Primers of qRT-PCR assay.

\begin{tabular}{ll}
\hline Name & Primer \\
\hline miR-451 & F: 5'-ACACTCCAGCTGGGAAACCGTTACCATTA -3' \\
& R: 5'-TGGTGTCGTGGAGTCG-3' \\
U6 & F: 5'-CTCGCTTCGGCAGCACA-3' \\
& R: 5'-AACGCTTCACGAATTTGCGT-3' \\
E-cadherin & F: 5'-CTGAGAACGAGGCTAACG-3' \\
& R: 5'-TTCACATCCAGCACATCC-3' \\
N-cadherin & F: 5'-CCACGCCGAGCCCCAGTATC-3' \\
& R: 5'-CCCCCAGTCGTTCAGGTAATCA-3' \\
\multirow{2}{*}{ Vimentin } & F: 5'-TTGAACGCAAAGTGGAATC-3' \\
& R: 5'-AGGTCAGGCTTGGAAACA-3' \\
c-Myc & F: 5'-GGAGGCTATTCTGCCCATTT-3' \\
& R: 5'-CGAGGTCATAGTTCCTGTTGGT-3' \\
GAPDH & F: 5'-TGGGTGTGAACCATGAGAAGT-3' \\
& R: 5'-TGAGTCCTTCCACGATACCAA-3' \\
\hline
\end{tabular}

of novel prognostic biomarkers and therapeutic targets for HCC.

Epithelial-to-mesenchymal transition (EMT) is considered as a key step of metastatic initiation of tumors, which has been reported to correlate with tumorigenesis, chemoresistance, especially invasion and metastasis $[4,5]$. An onset of typical EMT characteristic is come up with mesenchymal markers such as $\mathrm{N}$-cadherin, and Vimentin increased, while epithelial markers decreased simultaneously, which trigger disruption of cell-tocell adhesion. EMT occurs during HCC progression in response to early metastasis and invasion process, and HCCs with EMT characteristics consistently more venous invasion, metastases and a poor prognosis than those without EMT features [6, 7]. Thus, researches on EMT and its roles in HCC tumorigenesis and metastasis will provide a novel perspective from clinical and translational standpoints.

MicroRNAs (miRNAs) are a class of small noncoding RNAs that block translation or degradation of downstream target messenger RNAs by binding to the 3' untranslated region (3'-UTR) [8, 9]. Accumulating evidence indicates that miRNA dysfunction is implicated in proliferation, apoptosis, chemoradioresistance, and metastasis of tumors [10-12]. Recently, numerous miRNAs have been reported to be involved with $\mathrm{HCC}$ tumorigenesis, such as miR-221, 210, 29c, 100, 520e, 26a and 612 [13-19]. Previously, we have showed that miR-451 up-regulation inhibit growth and induce apoptosis in non-small cell lung cancer (NSCLC) cells. Importantly, restoration of miR-451 could reverse chemo- or radioresistance and EMT phenotypes of lung adenocarcinoma cells [20-22]. Although evidences suggesting miR-451 possibly involved in proliferation and migration of $\mathrm{HCC}[23,24]$, the clinicopathological and prognostic values of miR-451 and its roles in EMT and metastasis of HCC cells remain largely unclear. In this study, we clarified the significance of miR-451 in EMT and metastasis of HCC by using human tissue specimens, in vitro assays and animal models. We showed that reduced miR-451 was correlated with higher incidence of metastasis and poor survival of HCC patients. Restoration of miR-451 could reverse EMT and inhibit metastasis of HCC cells in vitro and in vivo. Furthermore, we testified that miR-451 exerted its anti-metastatic effects by directly targeting the oncogene c-Myc, which led to the activation of Erk1/2 signaling pathway. Our findings highlight the critical roles of miR-451 dysregulation in inhibiting metastasis of HCC through regulation of EMT process.

\section{RESULTS}

\section{Expression of miR-451 was inversely correlated with metastasis and prognosis in $\mathrm{HCC}$}

To explore the expression and significance of miR-451 in hepatocarcinogenesis, we first detected the expression of miR-451 in 20 paired of HCC and the adjacent nontumor tissues using qRT-PCR. The expression of miR-451 is significantly downregulated in HCC tissues when compared to the adjacent non-tumor tissues, and reduced miR-451 was observed to be significantly associated with advanced TNM stage, lymph node metastasis, vascular invasion and higher Edmondson grade in additional 88 HCC tissues (Figure $1 \mathrm{a}-1 \mathrm{~b}$ and Table 3 ). Furthermore, the Kaplan-Meier survival plots revealed an association of lower miR-451 expression levels with shorter disease-free survival (DFS) and overall survival 
(OS), and multivariate Cox regression analysis indicated that reduced miR-451 was an independent poor prognostic factor for HCC patients ( $P=0.009$; Figure 1c and Table 4). Then, we detected the expression levels of miR-451 in a panel of HCC cell lines with different metastatic potential (Figure 1d), and showed the expression of miR-451 was significantly lower in HCC cells when compared to normal human hepatocyte cell line L02, and the expression level $\mathbf{a}$

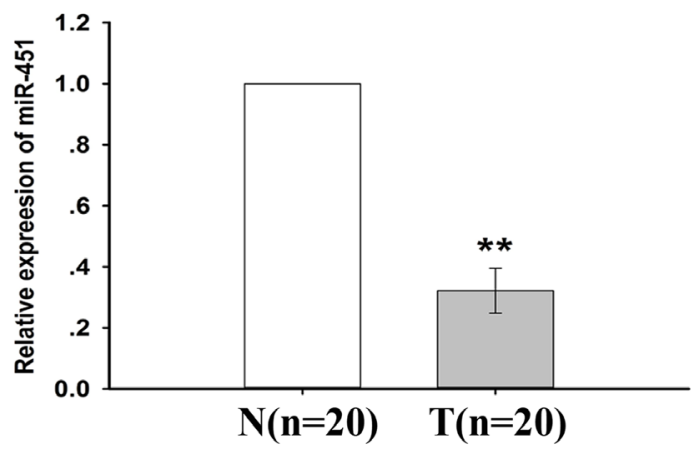

C
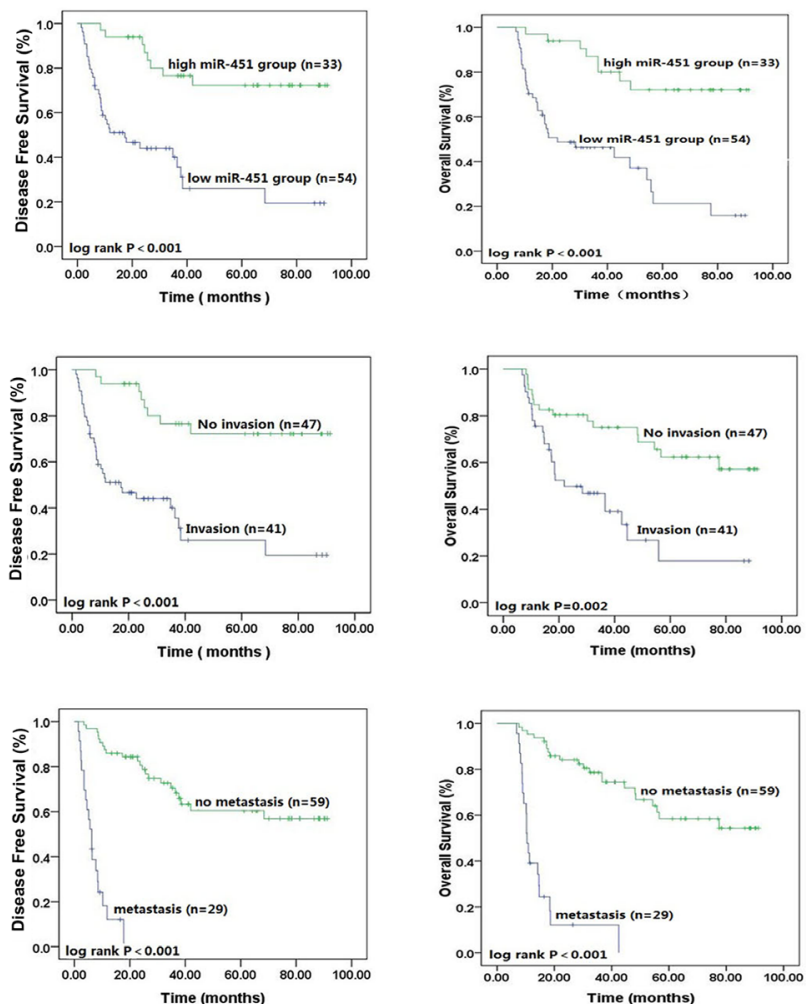

b
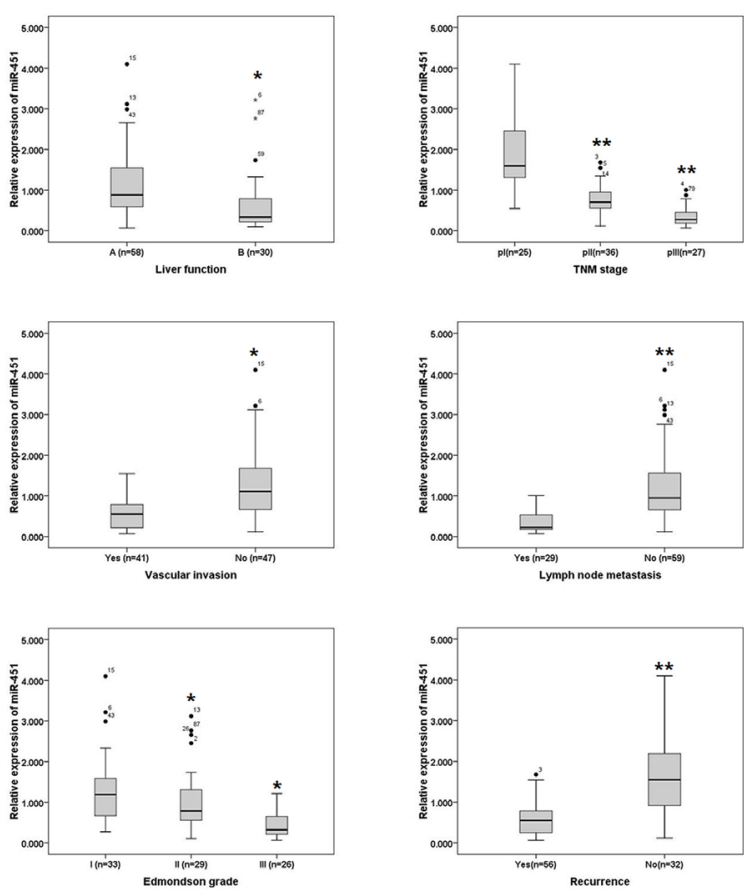

Figure 1: Expression of miR-451 in HCC cell lines and tissue samples. a. The relative expression level of miR-451 in 20 paired of HCC $(\mathrm{T})$ and the adjacent nontumor tissues $(\mathrm{N})$ was determined by qRT-PCR. The RNU6B small nuclear RNA was used as an internal control and the fold change was calculated by $2^{-\Delta \Delta \mathrm{Ct}}$. Cut off value $(0.972)$ was verified by ROC curve. b. The lower miR-451 expression in $88 \mathrm{HCC}$ tissue sample was observed to be closely correlated with poorer liver function, advanced TNM stage, higher edmondson grade, higher incidence of vascular invasion or lymph node metastasis and recurrence of HCC patients. c. Kaplan-Meier survival plots analysis of the association of miR-451 expression level, vascular invasion and lymph node metastasis with DFS and OS of HCC patients after curative resection. The survival data were compared with the log-rank test. d. qRT-PCR detection of relative miR-451 expression level in a normal human hepatocyte cell line (L02) and HCC cell lines (HepG2, SMMC7721, Bel7402, HCCLM3, MHCC97H) with different metastatic potentials. T: HCC tissues; N: the adjacent nontumor tissues. Results represent the average of three independent experiments (mean $\pm \mathrm{SD}) . * P<0.05$ and $* * P<0.01$. 
Table 2: Sequences of miRNAs or shRNAs used in the study.

\begin{tabular}{|c|c|}
\hline Name & Sequences \\
\hline miR-451 inhibitor & 5'-AACUCAGUAAUGGUAACGGUUU-3' \\
\hline \multirow[t]{2}{*}{ miR-451 mimics } & F 5'-AAACCGUUACCAUUACUGAGUU-3' \\
\hline & R 5'-CUCAGUAAUGGUAACGGUUUUU-3' \\
\hline \multirow[t]{2}{*}{ sh-control } & F 5'-CCGGGCTTCTCCGAACGTGTCACGTCTCGAGAAGAAACCAGTAAACGTAAGCTTTTTG-3' \\
\hline & R 5' - AATTCAAAAAGCTTCTCCGAACGTGTCACGTCTCGAGAAGAAACCAGTAAACGTAAGC-3' \\
\hline \multirow[t]{2}{*}{ sh-c-myc \#1 } & F 5'-CCGGCCCAAGGTAGTTATCCTTAAACTCGAGTTTAAGGATAACTACCTTGggTTTTTG-3' \\
\hline & R5' - AATTCAAAAACCCAAGGTAGTTATCCTTAAACTCGAGTTTAAGGATAACTACCTTGGG-3' \\
\hline \multirow[t]{2}{*}{ sh-c-myc \#2 } & F 5'- CCGGCAGTTGAAACACAAACTTGAACTCGAGTTCAAGTTTGTGTTTCAACtgTTTTTG-3' \\
\hline & R 5'- AATTCAAAAACAGTTGAAACACAAACTTGAACTCGAGTTCAAGTTTGTGTTTCAACTG-3' \\
\hline \multirow[t]{2}{*}{ sh-c-myc \#3 } & F 5'- CCGGCAGGAACTATGACCTCGACTACTCGAGTAGTCGAGGTCATAGTTCCtgTTTTTG-3' \\
\hline & R 5'- AATTCAAAAACAGGAACTATGACCTCGACTACTCGAGTAGTCGAGGTCATAGTTCCTG-3' \\
\hline
\end{tabular}

of miR-451 in the highly-metastatic HCC cell lines (HCCLM3 and MHCC97H) was much lower than those in the low-metastatic HCC cell lines (HepG2, SMMC7721, Bel7402), suggesting that miR-451 downregulation correlates with increased metastatic potential of $\mathrm{HCC}$ cells.

\section{Restoration of miR-451 significantly suppresses growth, migration and invasion of $\mathrm{HCC}$ cells in vitro}

To further determine the biological significance of miR-451 in HCC, we first performed gain of function experiment by stably transfecting an miR-451overexpressed plasmid (pcDNA/miR-451) into four human HCC cell lines (Figure 2a). Functional assays indicated that restoration of miR-451 could inhibit growth, induce $\mathrm{G}_{0} / \mathrm{G}_{1}$ arrest and increase apoptosis in HCC cells, which might correlate with a decrease in the cell anti-apoptotic ability (Bcl-2/Bax ratio) and a well know cellular $\mathrm{G}_{1} / \mathrm{S}$ transitional regulator (cyclinD1) (Supplementary Figure 1a-1d). Meanwhile, the effects of miR-451 on migration and invasion of HCC cells were further investigated. Wound scratch healing and matrigel transwell assays indicated that upregulation of miR-451 significantly inhibited migration and invasion of HCC cells (Figure 2b-2c). EMT, the sequence of events that converts adherent epithelial cells into migratory cells, is considered as a key step of metastatic initiation of tumor cells. Thus, we further determined the effects of miR-451 on EMT phenotypes of HCC cells. qRT-PCR and Western blotting assays showed that restoration of miR-451 in HCC cells induced the expression of epithelial markers (E-cadherin and $\beta$-catenin) that was accompanied by a concomitant decrease of mesenchymal markers (N-cadherin and Vimentin) (Figure 2d-2e). Likewise, immunofluorecence assay confirmed the expression of those protein markers in pcDNA/miR-451-transfected HCC cells (Supplementary Figure 2a-2b), which demonstrated re-acquisition of epithelial features and a loss of mesenchymal-like features in miR-451-upregulated HCC cells. Therefore, these results indicate that reduced miR-451 plays an important role in promoting $\mathrm{HCC}$ tumorigenesis, as well as induction of migration and invasion of $\mathrm{HCC}$ cells through regulation of EMT process.

\section{Restoration of miR-451 expression significantly inhibits in vivo tumorigenesis and metastasis of $\mathrm{HCC}$}

To confirm the above data in vivo, HepG2-miR-451 and Bel7402-miR-451 cells were injected subcutaneously into nude mice. Xenografts tumor volume was measured each week after palpable tumor formed, and mice were sacrificed 8 weeks after tumor implantation. The final tumor volume and weight of HepG2-miR-451 or Bel7402-miR-451 group were significantly smaller than that of HepG2-miR-NC or Bel7402-miR-NC group (Supplementary Figure 3a-3c). Also, qRT-PCR assay confirmed the upregulation of miR-451 and immunostaining assays revealed the increased positive rates of proliferating cell nuclear antigen (PCNA) and ki67, while TUNEL staining detection of apoptosis showed obvious nuclear fragmentation in HepG2-miR-451 or Bel7402-miR-451 group compared to the control groups (Supplementary Figure 3d-3f). Therefore, restoration of miR-451 could significantly inhibit tumorigenesis of HCC cells in vivo. 
Table 3: Correlations between miR-451 expression and clinicopathological factors of HCC patients.

\begin{tabular}{|c|c|c|c|c|}
\hline \multirow[b]{2}{*}{ Factors } & \multirow[b]{2}{*}{$\mathrm{n}$} & \multicolumn{2}{|c|}{ miR-451 expression } & \multirow[b]{2}{*}{$P$-value } \\
\hline & & low $(n=55)$ & $\operatorname{high}(n=33)$ & \\
\hline Gender & & & & 0.676 \\
\hline Male & 61 & 39 & 22 & \\
\hline Female & 27 & 16 & 11 & \\
\hline Age (years) & & & & 0.759 \\
\hline$>55$ & 49 & 36 & 20 & \\
\hline$\leq 55$ & 39 & 19 & 13 & \\
\hline Family history & & & & 0.526 \\
\hline Yes & 30 & 18 & 13 & \\
\hline No & 58 & 37 & 20 & \\
\hline Alcohol intake & & & & 0.648 \\
\hline No & 23 & 36 & 9 & \\
\hline Yes & 65 & 19 & 29 & \\
\hline HBV infection & & & & 0.310 \\
\hline Yes & 61 & 36 & 25 & \\
\hline No & 27 & 19 & 8 & \\
\hline Tumor diameter $(\mathrm{cm})$ & & & & 0.491 \\
\hline$\leq 5.0$ & 65 & 42 & 23 & \\
\hline$>5.0$ & 23 & 13 & 10 & \\
\hline Liver function & & & & 0.429 \\
\hline Child-Pugh A & 54 & 32 & 22 & \\
\hline Child-Pugh A & 34 & 23 & 11 & \\
\hline TNM stage & & & & $0.003^{*}$ \\
\hline I & 19 & 9 & 10 & \\
\hline II & 36 & 13 & 23 & \\
\hline III & 34 & 26 & 8 & \\
\hline Lymph node metastasis & & & & $0.022^{*}$ \\
\hline Yes & 32 & 25 & 7 & \\
\hline No & 56 & 30 & 26 & \\
\hline Vascular invasion & & & & $0.021^{*}$ \\
\hline Yes & 46 & 34 & 12 & \\
\hline No & 42 & 21 & 21 & \\
\hline Edmondson grade & & & & $0.038^{*}$ \\
\hline I & 29 & 13 & 16 & \\
\hline II & 29 & 19 & 10 & \\
\hline III & 30 & 23 & 7 & \\
\hline
\end{tabular}

Abbreviation: HCC: hepatocellular carcinoma; HBV: hepatitis B virus; ${ }^{*} P<0.05$. 
To further determine the effects of miR-451 on the in vivo tumor metastasis, the orthotopic HCC models were established using HCCLM3/miR-451 or MHCC97-H/ miR-451 cells with a microsyringe. After 8 weeks, mice were sacrificed, and their livers and lungs were isolated, immersed with neutral formalin and prepared for standard histological analysis (Figure 3a). The total incidence and number of lung metastatic nodules, as well as intrahepatic lesions in HCCLM3/miR-451 or MHCC97-H/miR-451 group were much lower than the control groups (Figure 3b-3c). Immunohistochemistry demonstrated the stronger staining of E-cadherin protein and weaker staining of Vimentin and MMP-2 proteins in HCCLM3/miR-451 or $\mathrm{MHCC} 97-\mathrm{H} / \mathrm{miR}-451$ group, when compared to the respective control group (Figure $3 \mathrm{~d}$ ). Collectively, restoration of miR-451 suppresses metastasis of $\mathrm{HCC}$ cells in vivo. $\mathbf{a}$

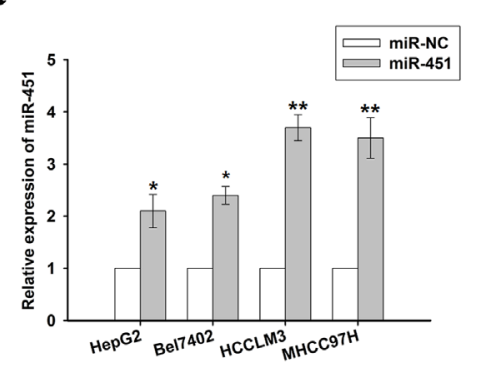

b
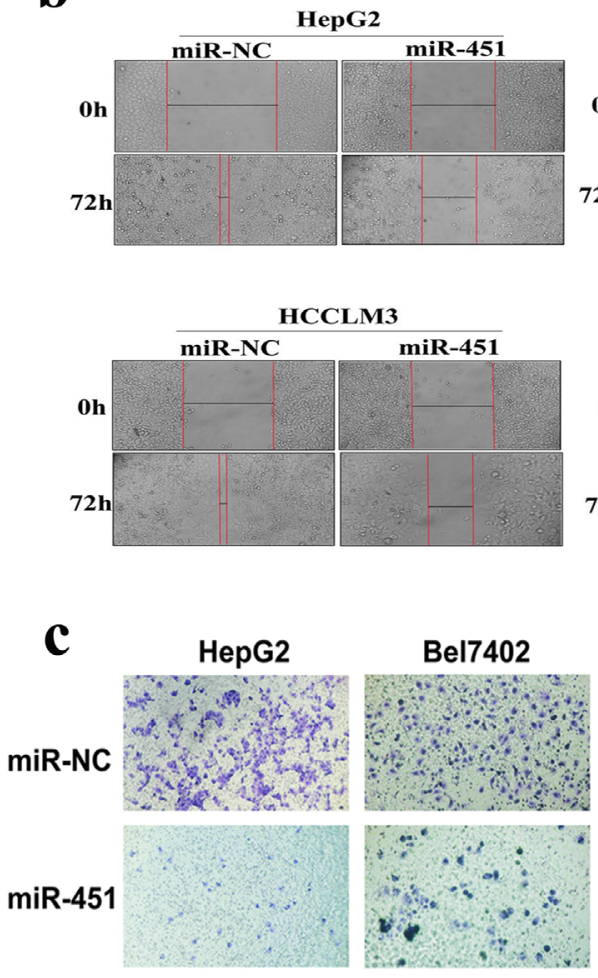

d
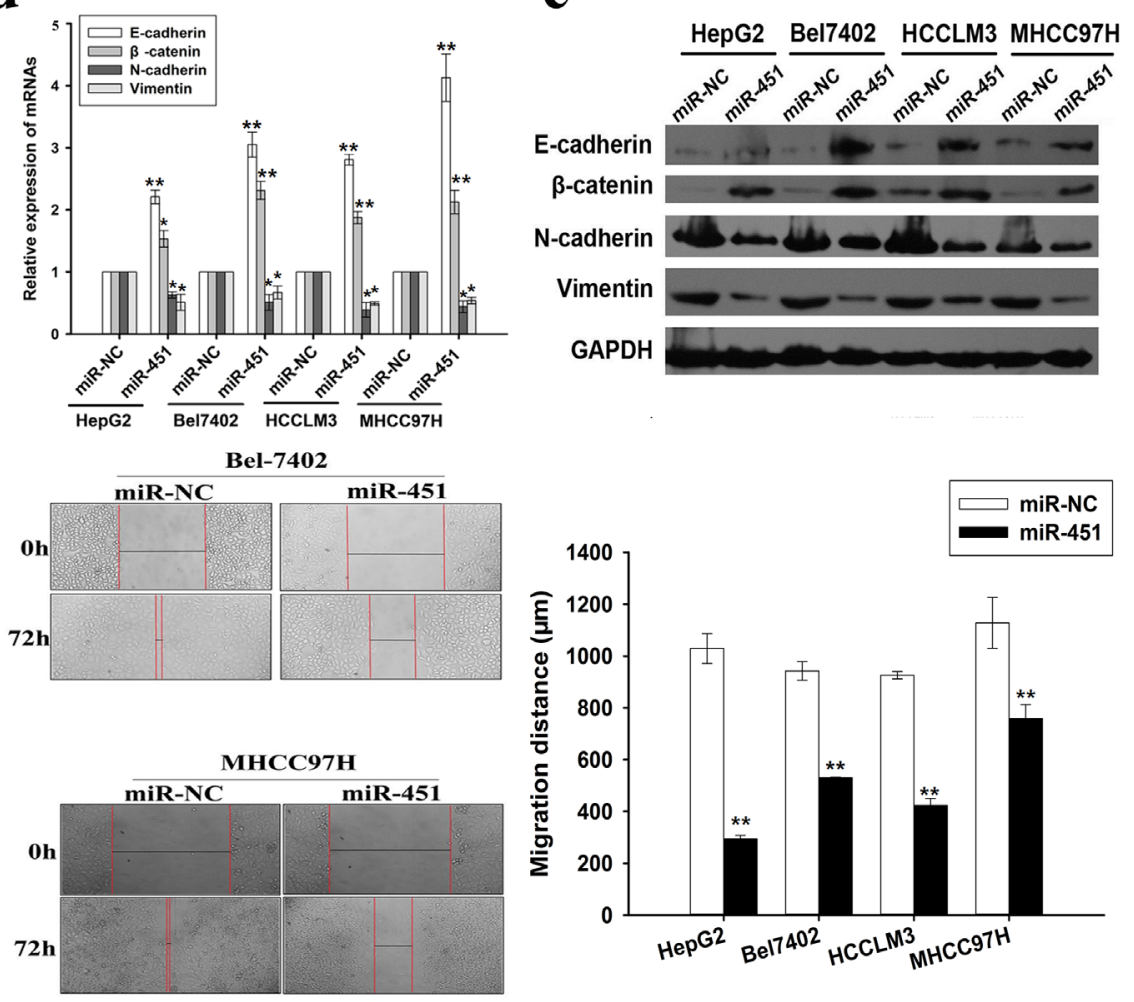

$\mathbf{e}$

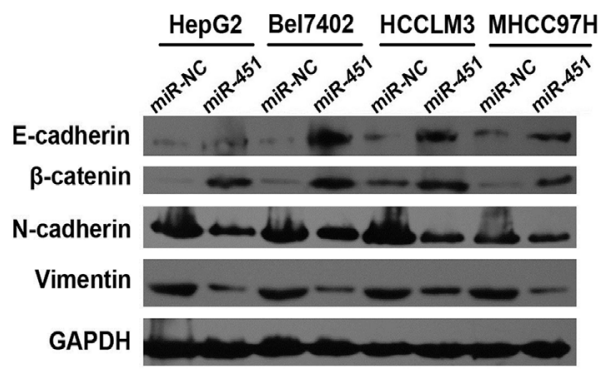

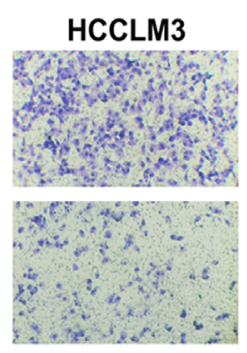
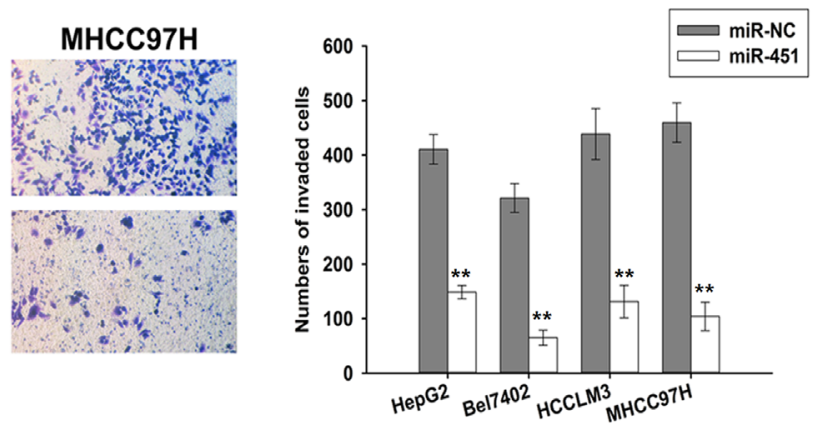

Figure 2: Re-expression of miR-451 negatively regulates HCC cell invasion and metastasis, as well as EMT process in vitro. a. qRT-PCR detection of miR-451 expression in HCC cells stably transfected with pcDNA/miR-451 or pcDNA/miR-NC. The RNU6B small nuclear RNA was used as an internal control. b. Wound scratch healing assay of HCC cell migration. A confluent monolayer of HCC cell stably expressing miR-451 or miR-NC was wounded. Photographs were taken immediately $(0 \mathrm{~h})$ and at $72 \mathrm{~h}$ after wounding, quantification of wound closure was done. c. Transwell invasion assay of HCC cells stably expressing miR-451 or miR-NC. Cells in five random fields of view at $100 \times$ magnification were counted and expressed as the average number of cells per field of view. d. qRT-PCR and e. Western blotting detection of mRNA and protein expression of epithelial markers (E-cadherin and $\beta$-catenin) and mesenchymal markers (N-cadherin and Vimentin) in HCC cells stably expressing miR-451 or miR-NC, respectively. GAPDH was used as an internal control. Results represent the average of three independent experiments (mean $\pm \mathrm{SD}$ ). ${ }^{*} P<0.05$ and $* * P<0.01$. 
Table 4: Univariate and multivariate analysis of prognostic variables by Cox regression analysis.

\begin{tabular}{|c|c|c|c|c|}
\hline \multirow{2}{*}{$\begin{array}{l}\text { Clinicopathological } \\
\text { factors }\end{array}$} & \multicolumn{2}{|c|}{ Univariate analysis } & \multicolumn{2}{|c|}{ Multivariate analysis } \\
\hline & $\mathrm{RR}(95 \% \mathrm{CI})$ & $P$-value & $\mathrm{RR}(95 \% \mathrm{CI})$ & $P$-value \\
\hline $\begin{array}{l}\text { TNM stage } \\
\text { (I/II/III) }\end{array}$ & $1.122(0.54-2.34)$ & $0.001 *$ & $34.1(8.51-136.7)$ & $0.001 *$ \\
\hline $\begin{array}{l}\text { Edmondson } \\
\operatorname{grade}(\mathrm{I} / \mathrm{II} / \mathrm{III})\end{array}$ & $1.198(0.98-4.04)$ & $0.02 *$ & $2.64(1.39-5.02)$ & $0.003^{*}$ \\
\hline $\begin{array}{l}\text { miR-451 expression } \\
\text { (low / high) }\end{array}$ & $1.52(1.08-2.12)$ & $0.016^{*}$ & $2.09(1.20-3.63)$ & $0.009 *$ \\
\hline
\end{tabular}

RR: relative ratio; 95\% CI: 95\% confidence interval.

\section{c-Myc was identified as a direct and functional target of miR-451 in HCC cells}

Previously, we have shown that miR-451 could reverse EMT phenotype of docetaxel-resistant lung adenocarcinoma cells by targeting the oncogene c-Myc. However, whether c-Myc was a functional target of miR451 in EMT and metastasis of HCC cells needs to be further elucidated. The complementary sequence of miR451 was exhibited in the 3'-UTR region of c-Myc (18911912 nt) (Supplementary Figure 4a), and we previously subcloned the 3'-UTR fragments of c-Myc, in which wildtype and mutant binding sites were harbored immediately downstream of the reporter gene (pLUC-c-Myc/3'UTR-wt and pLUC-c-Myc/3'-UTR-mut). Luciferase reporter analysis indicated that co-expression of miR-451 significantly reduced the activity of firefly luciferase that carried wild-type but not mutant 3'-UTR of c-Myc, while restoration of miR-451 could downregulate the expression of c-Myc protein in HCC cells and the both xenografts and orthotopic lung implanted model tumors of miR451-overexpressed HCC cells showed weaker staining of c-Myc protein (Supplementary Figure 4b-4d). These results demonstrate that miR-451 can negatively regulate the expression of c-Myc by directly targeting its 3'-UTR.

\section{Silencing of c-Myc mimics the effects of miR-451 on phenotypes of $\mathrm{HCC}$ cells}

To further explore the role of c-Myc in miR-451mediated EMT and metastasis of HCC cells, we first determine whether knockdown of c-Myc can mimic the

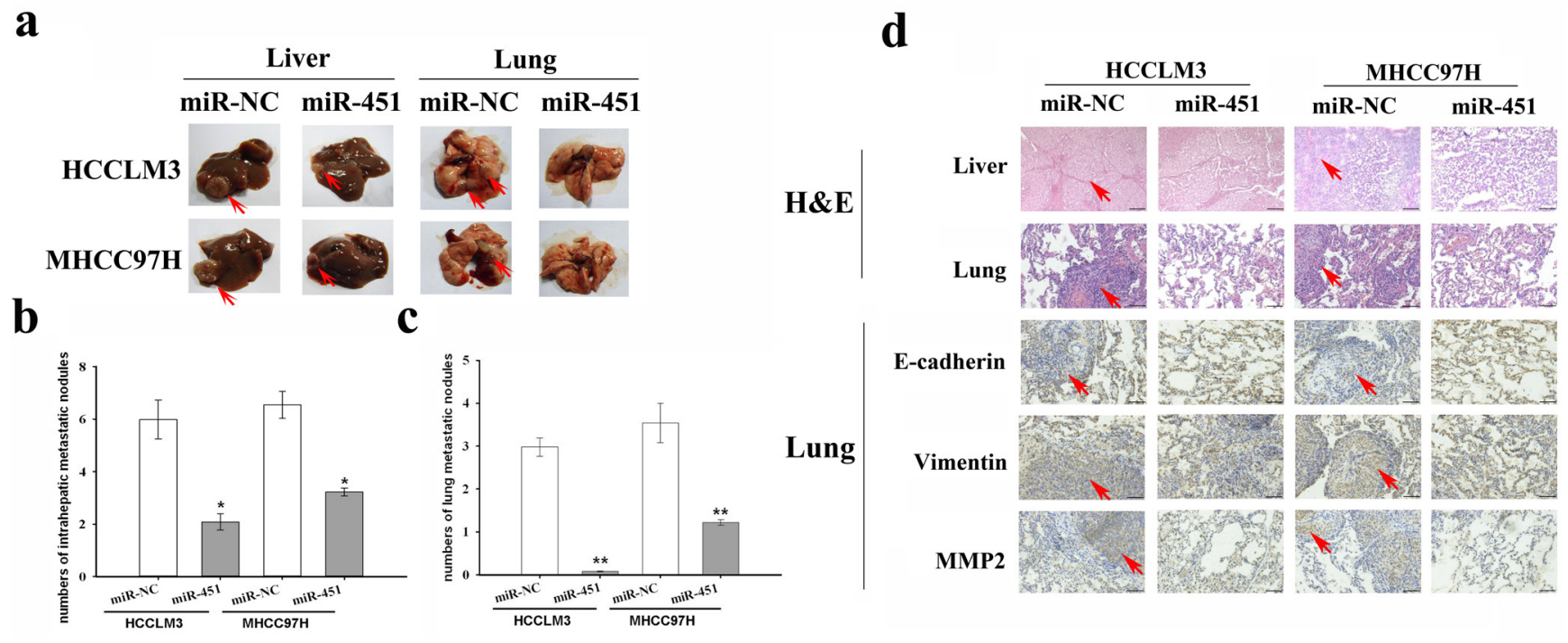

Figure 3: Re-expression of miR-451 inhibits metastasis of HCC cells in vivo. a. Liver primary lesion and lung metastasis as revealed by experimental nude mice (BALB/c nu/nu). Representative photos were shown above, respectively. b. The total incidence and number of intrahepatic metastatic nodules in the miR-451-overexpressed groups were much lower than the negative control. c. The number of lung metastatic nodules in HCCLM3/miR-451 or MHCC97H/miR-451 group was less than the control groups. d. Immunohistochemistry was performed to detect the expression of downstream targets and EMT markers. Vimenin and MMP-2 were dramatically decreased in pcDNA/miR-451 transfected groups, while E-cadherin was enhanced. Results represent the average of three independent experiments (mean \pm SD). $* P<0.05$ and $* * P<0.01$. Scale bar: $200 \mu \mathrm{m}$. 
effects of miR-451. Three short hairpin RNAs (pSil/shcMyc \#1, 2 or 3) were stably transfected into HCC cells, qRT-PCR and Western blotting confirmed that pSil/shcMyc3 had the best silencing effect on c-Myc in HCC cells (Supplementary Figure 5a). Then, qRT-PCR and Western blotting assays indicated pSil/shc-Myc\#3 could lead to the increased expression of epithelial markers and the decreased expression of mesenchymal markers in HCC cells (Figure 4a-4b). Likewise, immunofluorecence confirmed the changes of EMT-related markers in pSil/ shc-Myc\#3-transfected HCC cells (Supplementary Figure 6). Furthermore, the capacities of migration and invasion in pSil/shc-Myc\#3-transfected HCC cells were significantly reduced in comparison with those in pSil/ shcontrol-transfected cells (Figure 4c-4d). Therefore, silencing of c-Myc mimics the effects of miR-451 restoration in HCC cells. $\mathbf{a}$

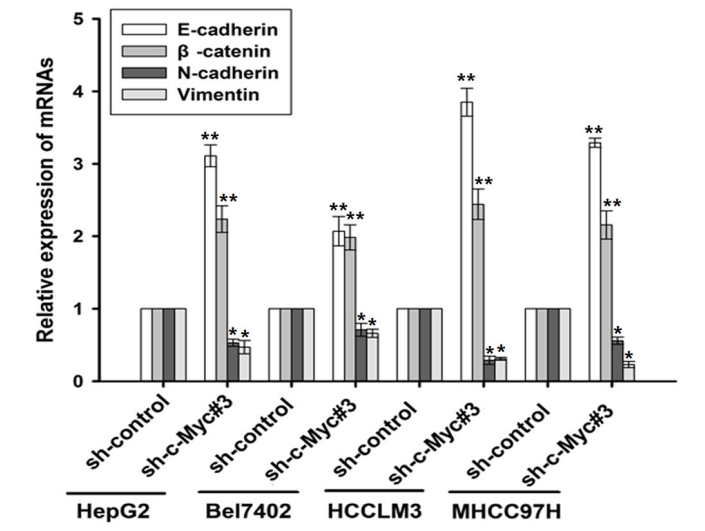

C
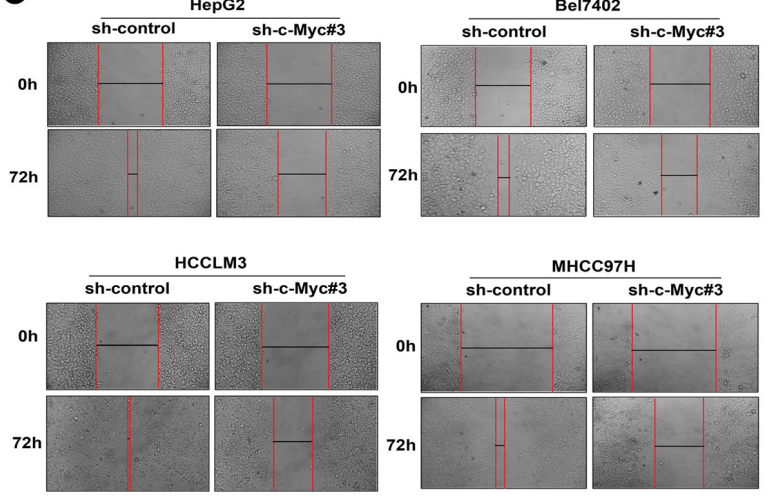

d

d HepG2

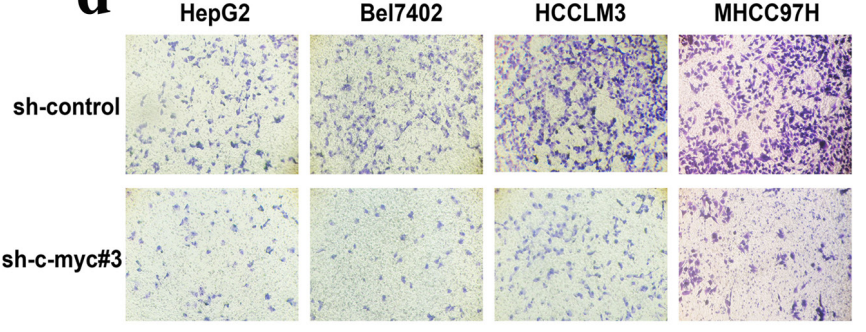

b

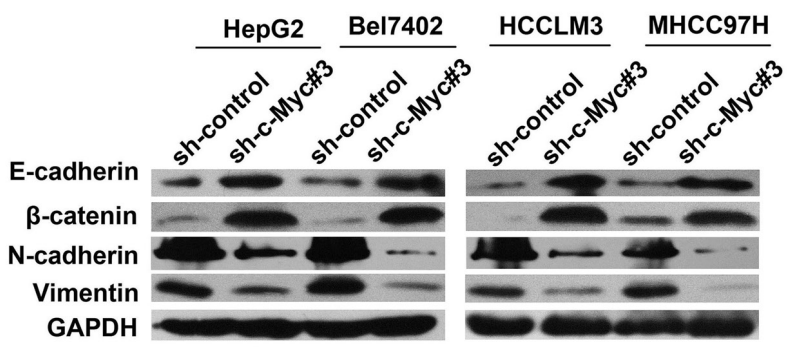

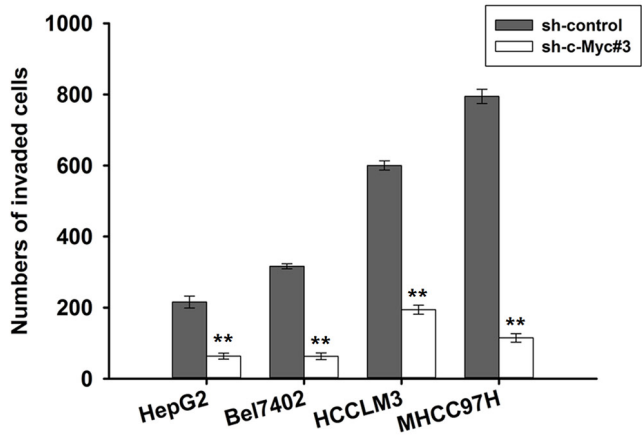

Figure 4: Effects of c-Myc downregulation on EMT, migration and invasion of HCC cells. a. qRT-PCR and b. Western blotting detection of the mRNA and protein expression of epithelial markers (E-cadherin and $\beta$-catenin) and mesenchymal markers (N-cadherin and Vimentin) in HCC cells stably expressing sh-c-Myc\#3 or sh-control, respectively. GAPDH was used as an internal control. c. Wound scratch healing assay of HCC cell migration. A confluent monolayer of HCC cells stably expressing sh-c-Myc\#3 or sh-control was wounded. Photographs were taken immediately $(0 \mathrm{~h})$ and at $72 \mathrm{~h}$ after wounding, quantification of wound closure was done. d. Transwell invasion assay of HCC cells stably expressing sh-c-Myc\#3 or sh-control. Cells in five random fields of view at $100 \times$ magnification were counted and expressed as the average number of cells per field of view. Results represent the average of three independent experiments (mean \pm SD). $* P<0.05$ and $* * P<0.01$. 


\section{Restoration of c-Myc reverses the effects of miR- 451 on phenotypes of $\mathrm{HCC}$ cells}

We then determined whether restoration of c-Myc could reverse the effects of miR-451 upregulation on EMT and metastasis of HCC cells. The plasmid vector overexpressing c-Myc was stably transfected into HCC cells, and Western blotting confirmed the significant upregulation of c-Myc protein (Supplementary Figure $5 b)$. It was also observed that the co-transfection of pcDNA3/c-Myc and pcDNA/miR-451 could reverse not only the decreased expression of c-Myc and mesenchymal markers but also the increased expression of epithelial markers in HCCLM3 and MHCC97H cells induced by miR-451 upregulation (Figure 5a-5b). Furthermore, the co-transfection could partially reverse the decreased capacities of migration and invasion in HCC cells induced by miR-451 upregulation (Figure $5 \mathrm{c}-5 \mathrm{~d}$ ). These data indicate that overexpression of c-Myc partially reverse the effects of miR-451 upregulation in HCC cells. $\mathbf{a}$

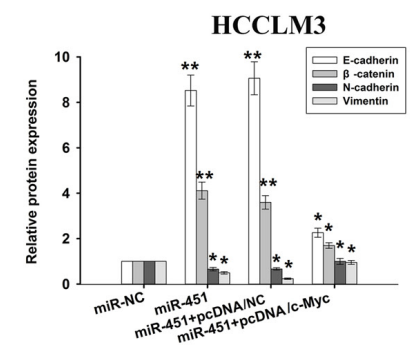

c

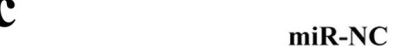

HCCLM3
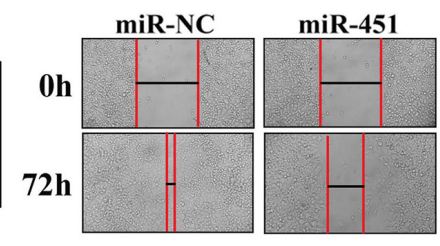

miR-451

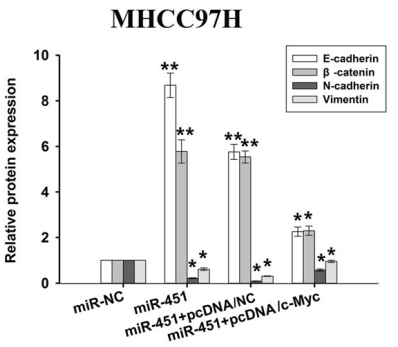

b

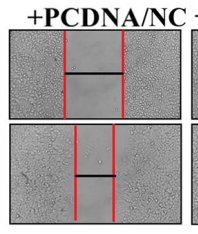

miR-451
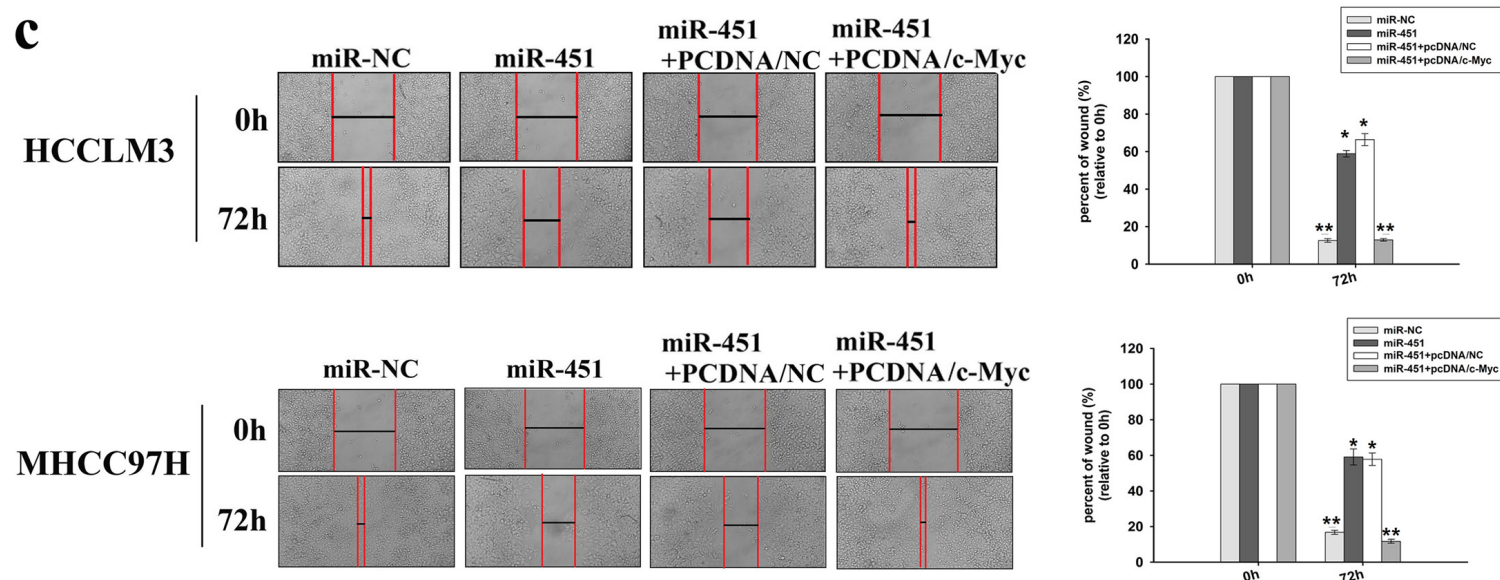

miR-451
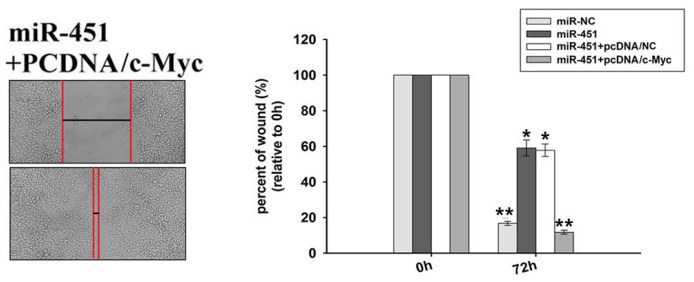

d

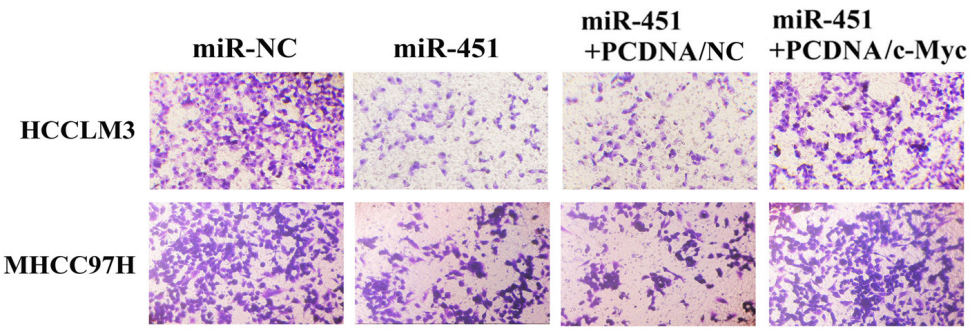

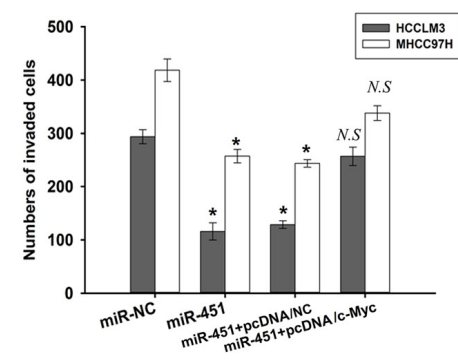

Figure 5: Overexpression of c-Myc reverses the effects of miR-451 upregulation on EMT, migration and invasion of HCC cells. a. qRT-PCR and $\mathbf{b}$. Western blotting detection of the mRNA and protein expression of epithelial markers (E-cadherin and $\beta$-catenin) and mesenchymal markers (N-cadherin and Vimentin) in HCC cells stably expressing miR-451 (or miR-NC) or HCC cells stably co-transfected with pcDNA/miR-451 and pcDNA/c-Myc (or pcDNA/NC). GAPDH was used as an internal control. c. Wound scratch healing assay of HCC cell migration. A confluent monolayer of HCC cells stably expressing miR-451 (or miR-NC) or HCC cells stably transfected with pcDNA/miR-451 and pcDNA/c-Myc (or pcDNA/NC). d. Transwell invasion assay of HCC cells stably expressing miR451 (or miR-NC) or HCC cells stably co-transfected with pcDNA/miR-451 and pcDNA/c-Myc (or pcDNA/NC). Cells in five random fields of view at $100 \times$ magnification were counted and expressed as the average number of cells per field of view. Results represent the average of three independent experiments (mean $\pm \mathrm{SD}$ ). ${ }^{*} P<0.05$ and ${ }^{* *} P<0.01 ;$ N.S, $P>0.05$. 

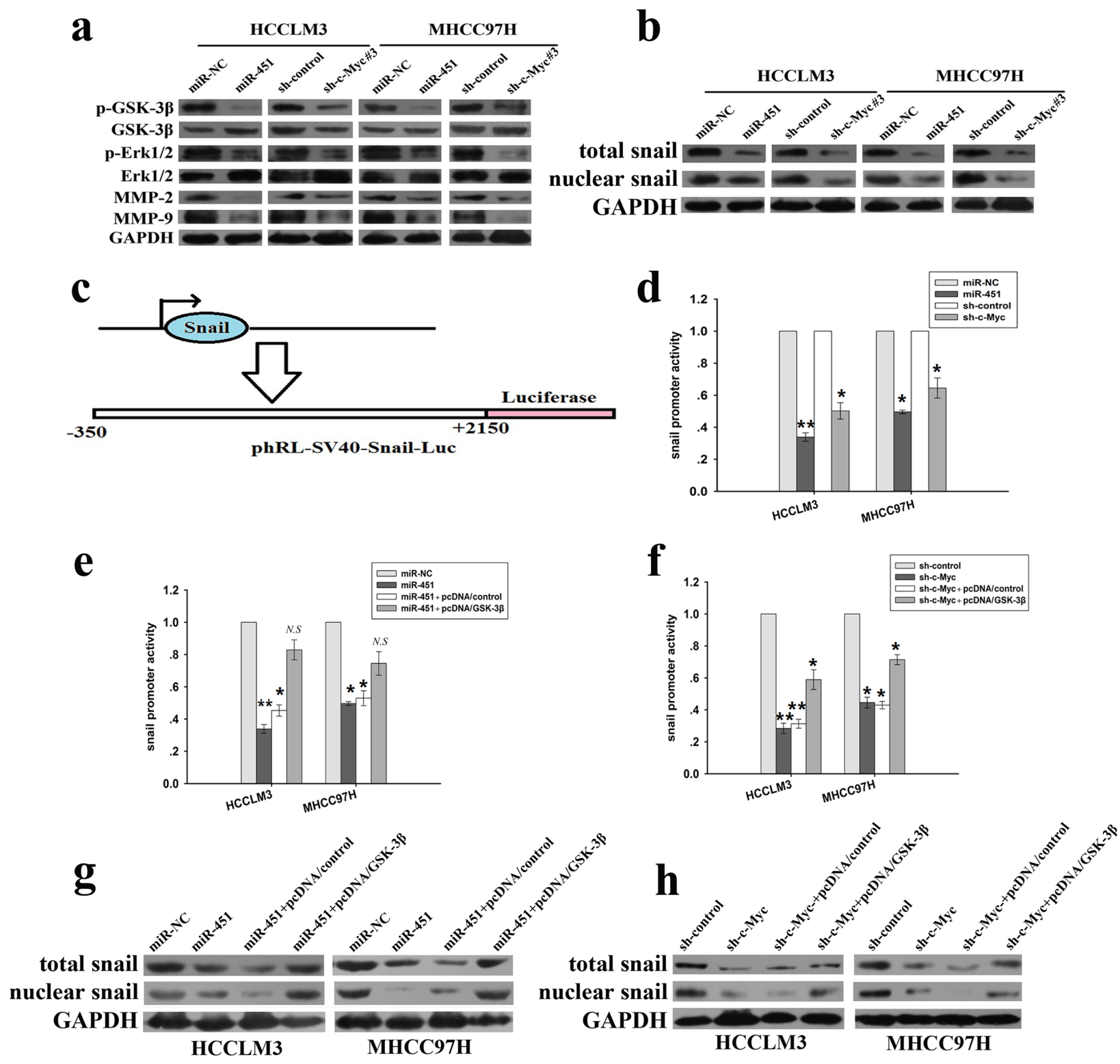

Figure 6: miR-451 regulates the expression of EMT-related markers and members of MMPs family through activation of Erk1/2 signaling in HCC cells by targeting c-Myc. a. Western blotting detection of the protein expression of total or phosphorylated

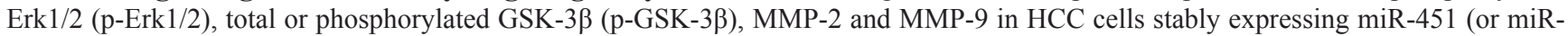
$\mathrm{NC}$ ) or HCC cells stably expressing sh-c-Myc\#3 (or sh-control), respectively. b. Western blotting detection of the protein expression of total or nuclear snail in HCC cells stably expressing miR-451 (or miR-NC) or HCC cells stably expressing sh-c-Myc\#3 (or sh-control), respectively. c. Schematic model of snail promoter/Luc plasmid. The luciferase reporter containing human snail promoter region $2500 \mathrm{bp}$ from transcription start site were constructed to confirmed the activity of snail promoter. $\mathbf{d}$. The histogram of snail promoter activity in HCC cells stably expressing miR-451 (or miR-NC) or sh-c-Myc\#3 (or sh-control). Each cell type was transiently transfected with $-2.5 \mathrm{~kb}$ snail promoter/Luc plasmid. Dual-luciferase reporter assays were performed on the lysed cells co-transfected with snail promoter/Luc (firefly luciferase) and phRL-SV (hRenilla luciferase) 24h after co-transfection. Reporter gene activation was determined as a relative ratio of firefly luciferase to hRenilla luciferase activity. e. As shown above, the histogram of snail promoter activity in HCC cells stably transfected with pcDNA/miR-451 (or pcDNA/miR-NC) or stably co-transfected with pcDNA/miR-451 and pcDNA/GSK-3 3 (or pcDNA/control). f. As shown above, the histogram of snail promoter activity in HCC cells stably transfected with $\mathrm{pSil} / \mathrm{sh}-\mathrm{c}-\mathrm{Myc} \# 3$ (or pSil/sh-control) or stably co-transfected with pSil/sh-c-Myc\#3 and pcDNA/GSK-3 $\beta$ (or pcDNA/NC). g. Western blotting detection of the protein expression of total or nuclear snail in HCC cells stably transfected with pcDNA/miR-451 (or pcDNA/miR-NC) or stably co-transfected with pcDNA/ miR-451 and pcDNA/GSK-3 $\beta$ (or pcDNA/NC). h. Western blotting detection of the protein expression of total or nuclear snail in HCC

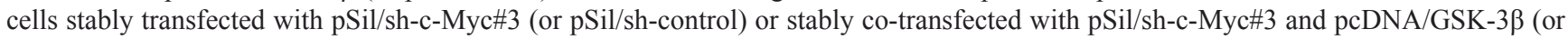
pcDNA/NC). GAPDH was used as an internal control. Results represent the average of three independent experiments (mean $\pm \mathrm{SD}) .{ }^{*} P<$ 0.05 and ${ }^{* *} P<0.01 ;$ N.S, $P>0.05$. 


\section{MiR-451 regulates the expression of EMT-related markers and members of MMPs family through activation of Erk1/2 signaling in $\mathrm{HCC}$ through targeting c-Myc}

Previously, overexpression of c-Myc has been reported to induce EMT in mammary epithelial cells via ERK-dependent GSK-3 $\beta$ inactivation and subsequent snail activation and promote tumor cell invasion via activation of MEK-ERK signaling as well as MMPs expression $[25,26]$. Therefore, we wondered whether miR-451 participates in the metastasis of HCC through regulation of EMT process by activating ERK signaling. Results of Western blotting showed the decreased expression level of phosphorylated Erk1/2 (p-Erk1/2), with downregulation of MMP-2 and MMP-9 proteins in pcDNA/miR-451 or pSil/shc-Myc\#3-transfected HCCLM3 and MHCC97H cells compared to the control cells, but no changes in the expression of total Erk1/2 (Figure 6a). Snail protein levels and its repressor functions are reported to be regulated by GSK- $3 \beta$ and the activity of GSK- $3 \beta$ enzyme is correlated with its Ser-9 phosphorylation level [27]. Here, both restoration of miR-451 and c-Myc downregulation could induce the decreased expression of p-GSK-3 $\beta$ (Ser-9) protein, but no changes of total GSK-3 $\beta$ protein (Figure 6a). Snail, a zinc finger family of transcriptional repressors, functions as a potent repressor of E-cadherin, and GSK-3 $\beta$ inhibition induces stability and increased nuclear levels of snail protein [28]. Thus, we will analyze whether miR-451/c-Myc affects the expression of snail protein and activity of its promoter. It was observed that both miR-451 upregulation and c-Myc downregulation induced the decreased expression of total and nuclear snail protein (Figure 6b). The luciferase reporter containing human snail promoter regions (2500 bp) from transcription start site were previously constructed to confirm the activity of snail promoter, and luciferase reporter analysis indicated that the transcriptional activity of snail promoter in both miR-451-upregulating and c-Myc-downregulating HCC cells was significantly inhibited (Figure 6c-6d). To further determine the roles of GSK-3 $\beta$ on the activity of snail promoter and the expression of snail protein, pcDNA/GSK- $3 \beta$ vector was previously constructed and the co-transfection of pcDNA/GSK-3 $\beta$ with pcDNA/ miR-451 or $\mathrm{pSil} / \mathrm{shc}-\mathrm{Myc} \# 3$ could partially restore the decreased activity of snail promoter and expression of total or nuclear snail protein in HCC cells induced by
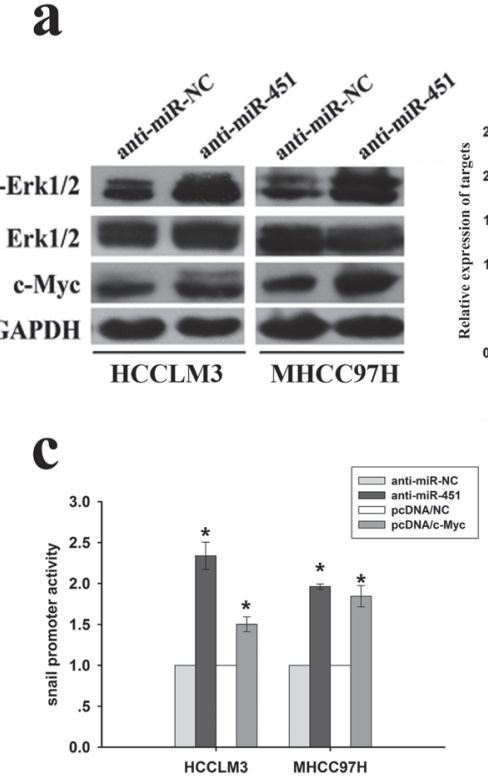

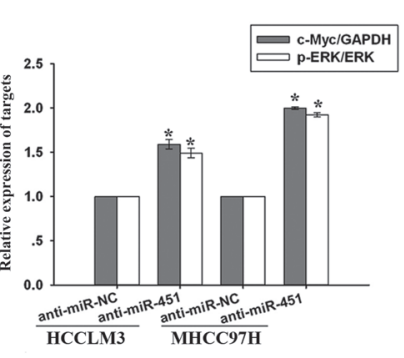

d

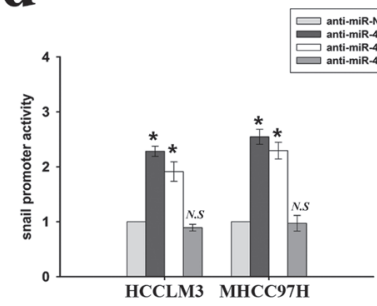

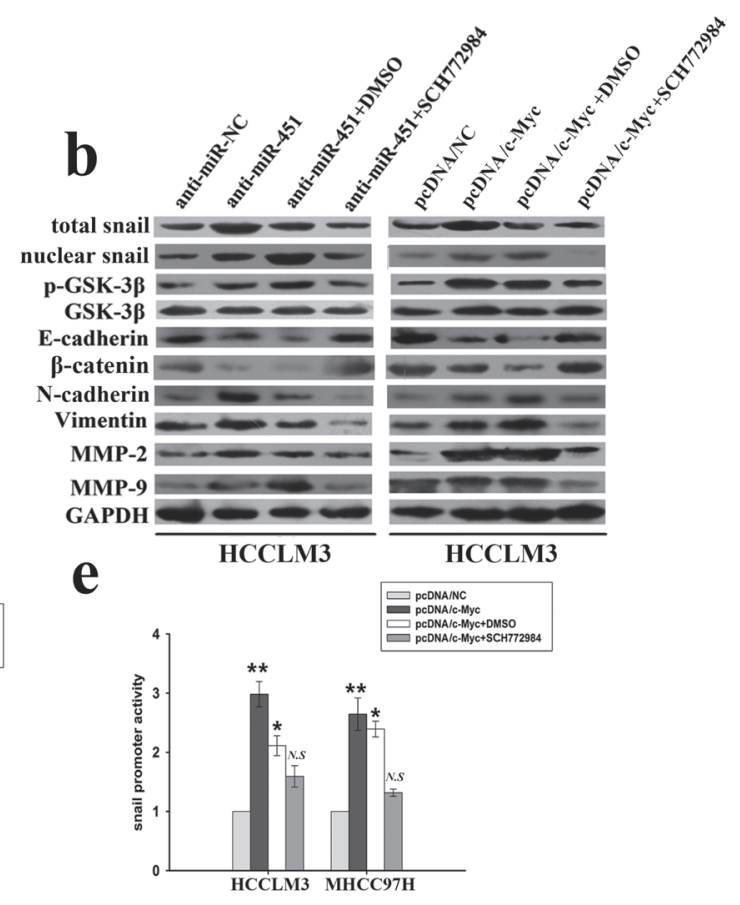

Figure 7: Role of ERK signaling in the effects of miR-451/c-Myc expression on snail or MMPs expression in HCC cells. a. Western blotting detection of the protein expression of c-Myc, p-Erk1/2 and total Erk1/2 in HCC cells transiently transfected with anti-miR-451 or anti-miR-NC, respectively. b. Western blotting detection of the protein expression of total or nuclear snail, total GSK$3 \beta$ or p-GSK-3 $\beta$, E-cadherin, $\beta$-catenin, N-cadherin, Vimentin, MMP-2 and MMP-9 in anti-miR-451 or pcDNA/c-Myc-transfected HCC cells treated with DMSO or SCH772984 (1.0 $\mu \mathrm{mol} / \mathrm{L}$, a special ERK inhibitor). c. The histogram of snail promoter activity in HCC cells transiently transfected with anti-miR-451 (or anti-miR-NC) or stably transfected with pcDNA/c-Myc (or pcDNA/control), respectively. d. The histogram of snail promoter activity in anti-miR-451 (or anti-miR-NC)-transfected HCC cells or anti-miR-451-transfected HCC cells treated with DMSO or SCH772984 (1.0 $\mu \mathrm{mol} / \mathrm{L})$. e. The histogram of snail promoter activity in pcDNA/c-Myc (or pcDNA/NC)-transfected HCC cells or pcDNA/c-Myc-transfected HCC cells treated with DMSO or SCH772984 $(1.0 \mu \mathrm{mol} / \mathrm{L})$. GAPDH was used as an internal control. Results represent the average of three independent experiments (mean $\pm \mathrm{SD}$ ). ${ }^{*} P<0.05$ and ${ }^{*} P<0.01 ;$ N.S, $P>0.05$. 
miR-451 upregulation or c-Myc downregulation (Figure 6e-6f). At the same time, transfection of anti-miR-451 could induce the increased expression of c-Myc and p-Erk1/2 proteins in HCCLM3 and MHCC97H cells (Figure 7a-7b). Also, miR-451 downregulation could lead to the decreased expression of epithelial markers and the increased expression of mesenchymal markers, total and nuclear Snail, p-GSK-3 $\beta$, MMP-2 and MMP9 proteins in above HCC cells, while both miR-451 downregulation and c-Myc overexpression could lead to the increased activity of snail promoter (Figure 7c-7d). Importantly, the increased activity of snail promoter in HCCLM3 cells induced by miR-451 downregulation or c-Myc overexpression could be both partially reversed by SCH772984 (a special ERK inhibitor, $1.0 \mu \mathrm{mol} / \mathrm{L}$ ) (Figure 7e-7f). Meanwhile, the increased expression of those proteins (total and nuclear snail, p-GSK-3 $\beta$, $\mathrm{N}$-cadherin and Vimentin, MMP-2 and MMP-9) and the decreased expression of E-cadherin and $\beta$-catenin in antimiR-451 or pcDNA/c-Myc-transfected HCCLM3 cells could be also partially reversed by SCH772984 (Figure 7c). These results clearly demonstrate that activation of Erk1/2 signaling mediates miR-451/c-Myc-induced EMT and metastasis in HCC cells by regulating the expression of EMT-related markers and members of MMPs family.

\section{Upregulation of c-Myc in HCC tissues, inversely correlated with miR-451 expression, correlates with metastasis and poor survival of patients}

Next, we performed qRT-PCR to detect the expression of c-Myc mRNA in above 20 paired HCC and the adjacent nontumor tissues, and showed that the relative expression level of c-Myc mRNA in HCC tissues was significantly higher than that in the matched adjacent nontumor tissues (Figure 8a), and a significant inverse correlation between the expression level of miR451 and that of c-Myc mRNA was observed in the 20 paired HCC tissues $(r=-0.776 ; P<0.001$; Figure $8 b)$. Likewise, Western blotting confirmed the high expression level of c-Myc protein in HCC tissues compared to the adjacent nontumor tissues (Figure 8c). Meanwhile, high c-Myc mRNA expression in HCC tissues was observed to significantly correlate with higher incidence of vascular invasion, lymph node metastasis and recurrence of patients (Figure 8d-8f). Furthermore, patients with high c-Myc expression showed much lower PFS or OS than those with low c-Myc expression ( $P=0.013$ or 0.005 ; Figure $8 \mathrm{~g}$ ). These results demonstrated that upregulation of c-Myc in HCC tissues inversely correlated with miR-451 expression and significantly correlated with metastasis and poor prognosis of patients.

\section{DISCUSSION}

Currently, more and more miRNAs were also converged to maintain distinctive characters of various processes, including tumor initiation, development as well as metastasis [29]. Previously, we have reported that miR451 inhibits growth and promotes apoptosis in NSCLC cells partially by targeting RAB14 [20]. Furthermore, we showed that miR-451 could reverse chemo- or radioresistance and EMT phenotypes of docetaxel-resistant lung adenocarcinoma cells by targeting c-Myc [21, 22]. Also, miR-451 is reported to be downregulated in many other human cancers, suggesting that miR-451 functions as a tumor suppressor [30]. Recently, the correlations of miR451 expression with HCC are also reported. Li' et al firstly reported that miR-451 inhibits cell proliferation in HCC through direct suppression of IKK- $\beta$ [23]. Also, Lv' et al reported that miR-451 regulates activating transcription factor 2 expression and inhibits HCC cell migration [24]. However, the clinicopathological and prognostic significance of miR-451 in HCC remains largely unclear. In this study, qRT-PCR testified that miR-451 was frequently downregulated in both HCC tissues and liver cancer cell lines. Interestingly, the expression of miR-451 in high-metastatic HCC cell lines was significantly lower than that in low-metastatic HCC cell lines. Also, reduced miR-451 expression in HCC tissues was observed to be closely with advanced TNM stage, lymph node metastasis, vascular invasion, edmondson grade and poor survival of patients, and multivariate analysis indicated that low miR451 expression was an independent poor prognostic factor for patients. These data demonstrated that reduced miR451 might play critical roles in HCC progression.

To further demonstrate the effects of miR-451 expression on malignant phenotypes of HCC cells, we then performed gain- and loss-of function experiments. It was observed that restoration of miR-451 could significantly inhibit in vitro growth and in vivo tumorigenesis of $\mathrm{HCC}$ cells by inducing $\mathrm{G}_{0} / \mathrm{G}_{1}$ arrest and apoptosis enhancement, which were consistent with the report by Li' et al [23]. Likewise, another report reveals a prominent role of miR451 as a tumor suppressor regulating HCC growth in a caspase-3-dependent manner [31]. Previously, we have shown that miR-451 significantly suppresses growth in NSCLC, and the tumor-suppressive effects of miR-451 are reported in other human cancers. For example, miR451 was reported to inhibit growth of colorectal carcinoma and glioma cells via targeting PI3k/Akt pathway $[32,33]$. Liu' et al showed that transfection of miR-451 mimics not only inhibited growth and invasion of tumor cells but also decreased angiogenic ability of HUVEC cell by targeting the IL6R pathway [34]. Interestingly, it was also reported that microRNA-451 regulates LKB1/AMPK signaling and allows adaptation to metabolic stress in glioma cells [35]. Under condition of glucose withdrawal, miR-451 downregulation increased cell survival and migration, 
a

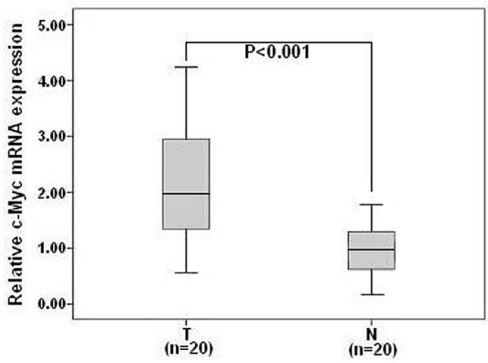

C

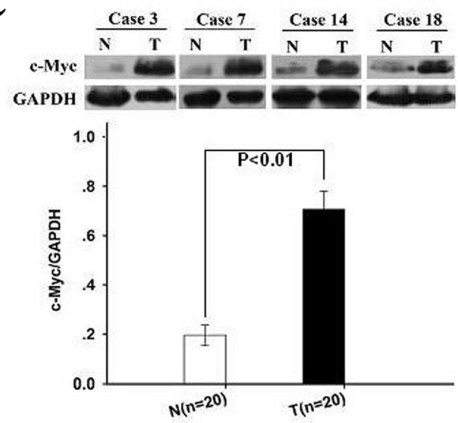

e

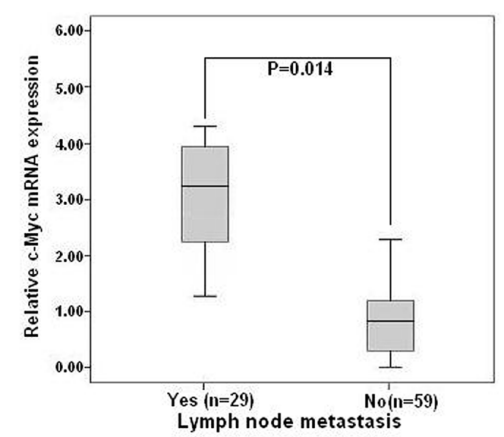

g

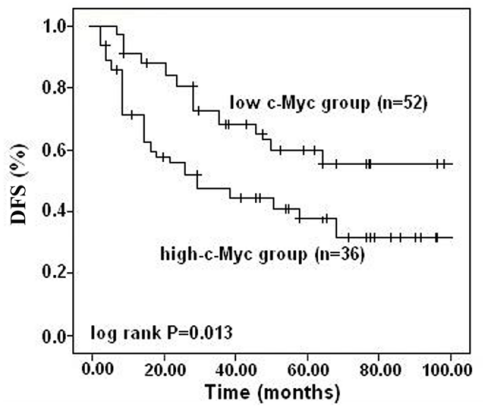

b

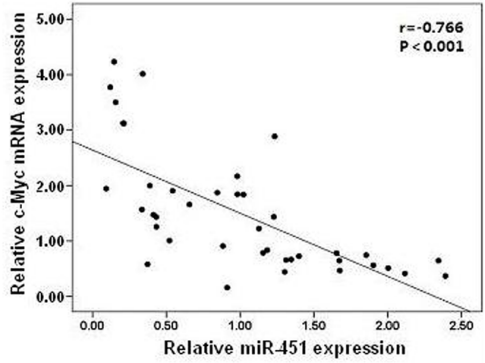

d

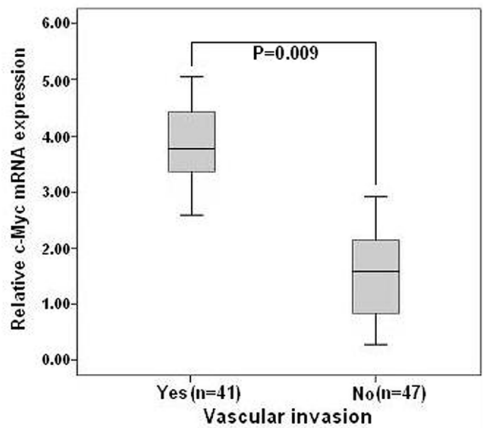

f
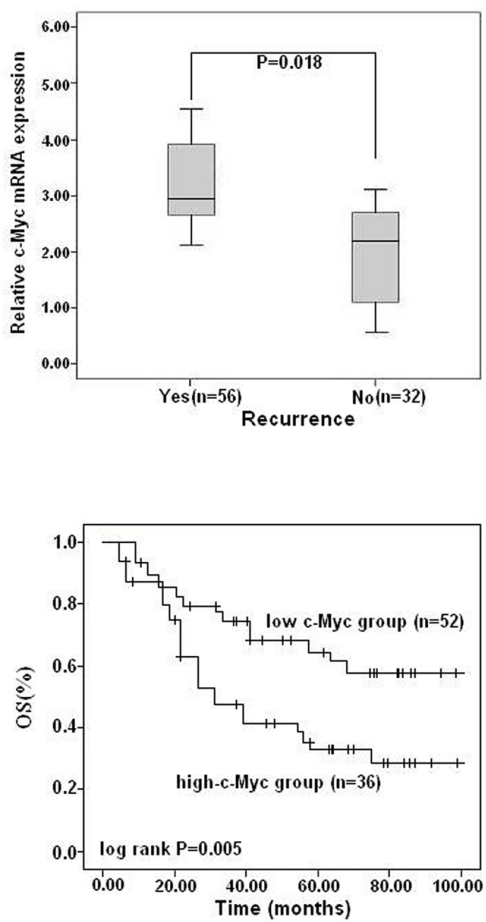

Figure 8: Upregulation of c-Myc in HCC tissues, inversely correlated with miR-451 expression, is associated with metastasis and poor survival of patients. a. qRT-PCR detection of relative c-Myc mRNA expression in 20 paired of HCC tissues $(\mathrm{T})$ and the adjacent nontumor tissues $(\mathrm{N})(P<0.001)$. b. Statistically significant inverse correlation between miR-451 and c-Myc mRNA expression in above tissues $(\mathrm{n}=40 ; \mathrm{r}=-0.766, P<0.001)$. $\mathbf{c}$. Western blotting detection of relative $\mathrm{c}-\mathrm{Myc}$ protein expression in above tissues $(P<0.01)$. d. qRT-PCR detection of relative c-Myc mRNA expression in HCC tissues with or without vascular invasion $(P=0.009)$. e. qRT-PCR detection of relative c-Myc mRNA expression in HCC tissues with or without lymph node metastasis $(P=0.014)$. f. qRT-PCR detection of relative c-Myc mRNA expression in $\mathrm{HCC}$ tissues with or without tumor recurrence $(P=0.018)$. g. The median value of c-Myc mRNA in all HCC tissues $(\mathrm{n}=88)$ was 2.08 and used as a cutoff value, and all patients were divided into two groups: high-c-Myc group $(\geq 2.18 ; \mathrm{n}=36)$ and low-c-Myc group $(<2.18 ; \mathrm{n}=52)$. Kaplan-Meier survival plots analysis of the association of c-Myc mRNA expression level with DFS $(P=0.013)$ and OS $(P=0.005)$ of HCC patients after curative resection. The survival data were compared with the log-rank test. GAPDH was used as an internal control. Results represent the average of three independent experiments (mean $\pm \mathrm{SD})$. 
suggesting that miR-451 might be a conditional switch controlling glioma cell proliferation and migration [36]. Our and other reports showed that upregulation of miR451 could reverse the resistance of lung adenocarcinoma cells to cisplatin and docetaxel [21, 37]. Additionally, miR-451 was reported to be involved in resistance of the MCF-7 breast cancer cells to chemotherapeutic drug doxorubicin, while tamoxifen downregulation of miR-451 increases $14-3-3 \zeta$ and promotes breast cancer cell survival and endocrine resistance [38, 39]. Lopotová and his colleagues testified the more complex relationship of miR451 and BCR-ABL, suggesting that miR-451 might be a putative predictor marker of Imatinib therapy response in chronic myeloid leukemia [40]. Collectively, these results clearly demonstrated that miR-451 functions as a tumor suppressor in human cancers.

EMT, the key process that drives metastasis, is characterized by loss of the epithelial marker, increased expression of the mesenchymal marker, and enhanced migratory and invasive behaviors [41]. Recently, the involvement of miRNAs in regulation of the EMT process of tumor cells was increasingly demonstrated. For example, ECM proteins and peptidases have been identified to be directly regulated by miR-200 family, which could alter the tumor microenvironment to restrain the EMT process in lung cancer [42]. In another study, EMT mediated by the transcriptional repressor snail 1 expression was regulated by miR-34 when wild-type p53 loss of function or mutation in multiple tumor cells [43]. Presently, only a few miRNAs are reported to be involved in regulation of EMT process in HCC cells. Zhang' et al showed that miR-148a suppresses EMT of hepatoma cells by targeting Met/Snail signaling [44]. Also, miR-491 is reported to be involved in metastasis of HCC by inhibitions of matrix metalloproteinase and EMT [45]. Conversely, other miRNAs promoting EMT of HCC cells are also found. For example, Overexpression of miR-106b could promote cell migration and metastasis in HCC by activating EMT process [46]. MiR216a/217-induced EMT targets PTEN and SMAD7 could promote drug resistance and recurrence of liver cancer [47]. However, the miRNA networks in EMT process of HCC remains largely unknown. Our previous study has shown that re-expression of miR-451 reverses EMT phenotype and inhibits metastasis of docetaxel-resistant lung cancer cells, but whether miR-451 plays important roles in formation of EMT phenotype in HCC cells is unknown. By qRT-PCR and Western blotting assays, we showed that re-expression of miR-451 could significantly downregulate the expression of mesenchymal markers (E-cadherin and $\beta$-catenin) and increase the expression of epithelial markers (N-cadherin and Vimentin) at both mRNA and protein levels, which were also confirmed by immunofluorecence assay. Furthermore, we showed that restoration of miR-451 could reduce the capacities of in vitro migration and invasion of HCC cells. More importantly, the in vivo metastasis of HCC cells was also inhibited by miR-451 upregulation. These above results demonstrated that restoration of miR-451 could reverse EMT phenotypes and suppress metastasis of HCC cells.

The important roles of miRNAs are to regulate the expression of their downstream specific targets through mRNA cleavage and / or by inhibition of translation. One miRNA could hold multiple target genes, effect gene could be repressed by multiple miRNAs [48]. Thus, to further explore the molecular mechanisms by which miR451 exerts its anti-metastatic functions, identification of its functional targets is essential. In our previous study, the pro-oncogene c-Myc was identified as a direct and functional target of miR-451 in lung cancer. c-Myc is a nuclear transcriptional factor and most of its targeting genes are crucial for ribosome biogenesis and protein synthesis, which are indispensable for cell proliferation and development [49]. It has been reported that c-Myc is highly expressed in many human cancers, including HCC [50]. Likewise, in $\mathrm{T}$ cell acute lymphoblastic leukemia (T-ALL), repression of tumor suppressor miR-451 is essential for NOTCH1-induced oncogenesis by directly targeting c-Myc [51]. In HCC, Li' et al showed that miR451 upregulation led to downregulation of cyclin D1 and c-Myc through inhibition of $\mathrm{NF}-\kappa \mathrm{B}$ pathway initiated by direct targeting of the IKBKB 3'-untranslated region [21], but it is still unclear whether miR-451 can directly regulate the expression of c-Myc through binding to the promoter region of c-Myc. To testify this, we first performed luciferase reporter assay, and showed that the luciferase activity could be reduced by miR-451. In addition, re-expression of miR-451 could downregulate the expression of c-Myc protein in HCC cells. More importantly, silencing of c-Myc could mimic the effects of miR-451 upregulation on malignant phenotypes of HCC cells, while overexpression of c-Myc could partially reverse the phenotypical changes of HCC cells induced by miR-451 upregulation. Furthermore, the expression of c-Myc in HCC tissues was significantly higher than that in the adjacent nontumor tissues, and statistically significant inverse association between miR-451 and c-Myc expression in HCC tissues was observed. These data clearly demonstrated that c-Myc was a direct and functional target of miR-451 in HCC. Subsequently, we further investigated the possible downstream signaling pathways. Although the precise role of c-Myc in metastasis process and EMT is still elusive, multiple studies have shown that c-Myc controls and supports this complex multistep process at different stages of human cancers [52]. Here, we showed that both miR451 upregulation and c-Myc downregulation induced the decreased expression of p-Erk1/2, p-GSK-3 $\beta$, MMP-2 and MMP-9 proteins, the decreased expression of total or nuclear snail proteins, and eventually the reduced activity of snail promoter. Importantly, overexpression of GSK$3 \beta$ could partially reverse the decreased activity of snail 
promoter reporter induced by miR-451 upregulation or c-Myc downregulation, while the ERK inhibitor could not only reverse the increased expression of $\mathrm{p}-\mathrm{GSK}-3 \beta$, total or nuclear snail, E-cadherin and MMPs in HCC cells induced by miR-451 downregulation, but also reverse the increased activity of snail promoter induced by miR-451 downregulation and c-Myc overexpression. These results clearly demonstrated that miR-451/c-Myc/Erk1/2/GSK-3 $\beta$ or MMPs signaling pathway might be involved in EMT and metastasis of HCC cells (Supplementary Figure 7).

In summary, we identified that reduced miR451 correlates with early recurrence, poor survival and metastasis of HCC patients. Re-expression of miR451 reduces tumorigenesis, reverses EMT and inhibits metastasis in HCC cells via inactivation of the Erk1/2 signaling pathway, at least partially by targeting c-Myc. Therefore, miR-451 may be a potential prognostic biomarker, and targeting this novel miR-451/c-Myc/Erk axis will be a promosing strategy for the treatment of metastatic HCCs.

\section{MATERIALS AND METHODS}

\section{Patients and tissue samples}

20 paired primary HCC and corresponding nontumor liver tissues, as well as 88 additional primary HCC surgical specimen were collected at the Department of Hepatobiliary Surgery of First Hospital Affiliated to the Chinese PLA General Hospital and the Liver Disease Center of the 81th Hospital of PLA from 2005 to 2007. The informed consent was signed from all the patients. None of preoperative treatment was done. International Union Against Cancer (UICC) TNM classification were used to verify the clinical stage. All clinical records were reviewed, and details were listed in Table 3 . The pathological judgment was determined by two senior pathologists with double-blind. All the cases were closely followed up for recurrence or death. The tissues were rapidly frozen and stored in liquid nitrogen. Scientific and ethic approval was certificated by the Ethics Committee of Jiangsu Province Medical Association.

\section{Cell culture}

Four human hepatoma cell lines (HepG2, Bel7402, HCCLM3, MHCC97H) and a normal human hepatocyte cell line (L02) were purchased from the Cell Bank of Shanghai institute of cell biology, Chinese Academy of Medical Sciences (Shanghai, China). All these cell lines were cultured in Dulbecco's modified Eagle's medium (DMEM) supplemented with 10\% fetal bovine serum (GIBCO), 100U/ml penicillin and 100ug/ml streptomycin at $37^{\circ} \mathrm{C}$ in a humidified atmosphere of $5 \% \mathrm{CO}_{2}$ and $95 \%$ air.

\section{Cell transfection}

The miR-451-overexpressing plasmid vector (pcDNA/miR-451) and GSK-3 $\beta$-overexpressing plasmid vector (pcDNA/GSK-3 $\beta$ ) were previously constructed and preserved in our lab. miR-451 inhibitor (antimiR-451) and mimics, as well as c-Myc short hairpin RNA (pSil/sh-c-Myc\#1, 2, 3), and nonspecific control shRNA (pSil/control) were all chemically synthesized by Genechem (Shanghai, China), as listed in Table.2. The stable transfection was performed using Lipofectamine 2000 (Invitrogen, Carlsbad, CA) according to the manufacturer's protocol as described previously [20].

\section{Quantitative real-time PCR (qRT-PCR) assay}

Real-time qRT-PCR analysis of mature miRNA and mRNA was performed using TaqMan ${ }^{\circledR}$ MicroRNA Cellsto-CT ${ }^{\text {TM }}$ Kit as described previously $[53,54]$.

\section{Western blotting assay}

The expression of E-cadherin, N-cadherin, Vimentin, $\beta$-catenin-c-Myc, cyclinD1, Bax, Bcl-2, NF- $\mathrm{B}$,

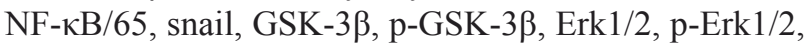
MMP-2, MMP-9 and GAPDH proteins was detected using Western blotting assay as previously described [21]. All bodies were purchased from Univ-bio Inc (Shanghai, China).

\section{Colony formation assay}

The cells were transplanted in a 6-well culture dish and maintained in DMEM containing 10\% FBS. The medium was renewed 24 hours later. After 2 weeks, cells were stained with $0.1 \%$ crystal violet after fixed with methanol. All the visible colonies were calculated manually.

\section{Flow cytometric analysis}

An annexin V-fluorescein isothiocyanate (FITC) apoptosis detection Kit and cell cycle detection Kit (KeyGEN Biotech) was used according to the manufacturer's instructions as previously described [20].

\section{In vitro migration and invasion assays}

The wound healing and transwell assays were performed as described previously [55]. 


\section{Immunofluorescence assay}

The expression E-cadherin, $\beta$-catenin, $N$-cadherin and Vimentin proteins in HCC cells was determined by an immunofluorescence assay as described previously [22].

\section{Immunohistochemistry assay}

Paraffin-embedded, formalin-fixed tissues were immunostained for E-cadherin, Vimentin, MMP-2, c-Myc, $\mathrm{Ki}-67, \mathrm{PCNA}$ proteins as previously described [20].

\section{In vivo tumor growth and metastasis assays}

Animal experiments were certificated by the Institutional Committee for Animal Research. Female athymic BALB/c nude mice (6 week old) were purchased from the Department of comparative medicine (Jinling Hospital, Nanjing, China). Subcutaneous xenografts or orthotopic metastatic model were establilshed as as previously described [55].

\section{Luciferase reporter assay}

Luciferase reporter containing wild type 3'-UTR of c-Myc (pLUC/c-Myc/3'-UTR-wt) and mutant reporter (pLUC/c-Myc/3'-UTR-mut) were previously established and preserved in our lab. The luciferase assay was performed as previously described [21,22].

\section{Promoter reported gene analysis}

The $-2.5 \mathrm{~kb}$ human snail promoter was cloned and insert in phRL-SV luciferase reporter vector which was purchased from Promega. Cor. (Madison, WI, USA). The promoter activity was detected by Dual-Luciferase Reporter Assay kit (Promega, USA) as previously described $[21,22]$.

\section{Statistical analysis}

All statistical analyses were performed with SPSS version 17.0 for Windows (SPSS Inc., Chicago, IL). Qualitative data were determined by descriptive statistics, including chi-square test or Fisher's exact test when appropriate. Survival analysis was estimated by KaplanMeier method. Cox proportional hazards regression was applied for multivariate analysis. $P<0.05$ was considered statistically significant.

\section{ACKNOWLEDGMENTS}

We thank Dr. Liu Biao for pathological analyses and Huo $\mathrm{Da}$ from the Nanjing University for animal model establishment. This work was supported by National Natural Science Foundation of China (No. 81402492, 81172335, 81272474 and 81301913) and Natural Science Foundation of Jiangsu province (BK2012371).

\section{CONFLICTS OF INTEREST}

There is no conflict of interest.

\section{REFERENCES}

1 Siegel R, Naishadham D, Jemal A. Cancer statistics, 2013. CA Cancer J Clin 2013; 63: 11-30.

2 Rampone B, Schiavone B, Martino A, Viviano C, Confuorto G. Current management strategy of hepatocellular carcinoma. World J Gastroenterol 2009; 15: 3210-3216.

3 Morise Z, Kawabe N, Tomishige H, Nagata H, Kawase J, Arakawa S, et al. Recent advances in the surgical treatment of hepatocellular carcinoma. World J Gastroenterol 2014; 20: 14381-14392.

4 Meng F, Wu G. The rejuvenated scenario of epithelialmesenchymal transition (EMT) and cancer metastasis. Cancer Metastasis Rev 2012; 31: 455-467.

5 Iwatsuki M, Mimori K, Yokobori T, Ishi H, Beppu T, Nakamori S, et al. Epithelial-mesenchymal transition in cancer development and its clinical significance. Cancer Sci 2010; 101: 293-299.

6 Ogunwobi OO, Liu C. Therapeutic and prognostic importance of epithelial-mesenchymal transition in liver cancers: insights from experimental models. Crit Rev Oncol Hematol 2012; 83: 319-328.

7 van Zijl F, Zulehner G, Petz M, Schneller D, Kornauth C, Hau M, et al. Epithelial-mesenchymal transition in hepatocellular carcinoma. Future Oncol 2009; 5: 11691179.

8 Sevignani C, Calin GA, Siracusa LD, Croce CM. Mammalian microRNAs: a small world for fine-tuning gene expression. Mamm Genome 2006; 17: 189-202.

9 Bartel DP. MicroRNAs: genomics, biogenesis, mechanism, and function. Cell 2004; 116: 281-297.

10 Shenouda SK, Alahari SK. MicroRNA function in cancer: oncogene or a tumor suppressor? Cancer Metastasis Rev 2009; 28: 369-378.

11 Cui SY, Wang R, Chen LB. MicroRNAs: key players of taxane resistance and their therapeutic potential in human cancers. J Cell Mol Med 2013; 17: 1207-1217.

12 Zhao L, Lu X, Cao Y. MicroRNA and signal transduction pathways in tumor radiation response. Cell Signal 2013; 25: 1625-1634.

13 Rong M, Chen G, Dang Y. Increased miR-221 expression 
in hepatocellular carcinoma tissues and its role in enhancing cell growth and inhibiting apoptosis in vitro. BMC Cancer 2013; 13: 21.

14 Ying Q, Liang L, Guo W, Zha R, Tian Q, Huang S, et al. Hypoxia-inducible microRNA-210 augments the metastatic potential of tumor cells by targeting vacuole membrane protein 1 in hepatocellular carcinoma. Hepatology 2011; 54: 2064-2075.

15 Bae HJ, Noh JH, Kim JK, Eun JW, Jung KH, Kim MG, et al. MicroRNA-29c functions as a tumor suppressor by direct targeting oncogenic SIRT1 in hepatocellular carcinoma. Oncogene 2014; 33: 2557-2567.

16 Chen P, Zhao X, Ma L. Downregulation of microRNA-100 correlates with tumor progression and poor prognosis in hepatocellular carcinoma. Mol Cell Biochem 2013; 383: 49-58.

17 Zhang S, Shan C, Kong G, Du Y, Ye L, Zhang X. MicroRNA-520e suppresses growth of hepatoma cells by targeting the NF- $\mathrm{kB}$-inducing kinase (NIK). Oncogene 2012; 31: 3607-3620.

18 Yang X, Liang L, Zhang XF, Jia HL, Qin Y, Zhu XC, et al. MicroRNA-26a suppresses tumor growth and metastasis of human hepatocellular carcinoma by targeting interleukin-6Stat3 pathway. Hepatology 2013; 58: 158-170.

19 Tao ZH, Wan JL, Zeng LY, Xie L, Sun HC, Qin LX, et al. miR-612 suppresses the invasive-metastatic cascade in hepatocellular carcinoma. J Exp Med 2013; 210: 789-803.

20 Wang R, Wang ZX, Yang JS, Pan X, De W, Chen LB. MicroRNA-451 functions as a tumor suppressor in human non-small cell lung cancer by targeting ras-related protein 14 (RAB14). Oncogene 2011; 30: 2644-2658.

21 Wang R, Chen DQ, Huang JY, Zhang K, Feng B, Pan $\mathrm{BZ}$, et al. Acquisition of radioresistance in docetaxelresistant human lung adenocarcinoma cells is linked with dysregulation of miR-451/c-Myc-survivin/rad-51 signaling. Oncotarget 2014; 5: 6113-6129.

22 Chen D, Huang J, Zhang K, Pan B, Chen J, De W, et al. MicroRNA-451 induces epithelial-mesenchymal transition in docetaxel-resistant lung adenocarcinoma cells by targeting proto-oncogene c-Myc. Eur J Cancer 2014 Oct 10. [Epub ahead of print]

23 Li HP, Zeng XC, Zhang B, Long JT, Zhou B, Tan GS, et al. miR-451 inhibits cell proliferation in human hepatocellular carcinoma through direct suppression of IKK- $\beta$. Carcinogenesis 2013; 34: 2443-2451.

24 Lv G, Hu Z, Tie Y, Du J, Fu H, Gao X, et al. MicroRNA-451 regulates activating transcription factor 2 expression and inhibits liver cancer cell migration. Oncol Rep 2014; 32: 1021-1028.

25 Cho KB, Cho MK, Lee WY, Kang KW. Overexpression of c-myc induces epithelial mesenchymal transition in mammary epithelial cells. Cancer Lett 2010; 293: 230-239.

26 Han G, Wang Y, Bi W. C-Myc overexpression promotes osteosarcoma cell invasion via activation of MEK-ERK pathway. Oncol Res 2012; 20: 149-156.

27 Ding Q, He X, Xia W, Hsu JM, Chen CT, Li LY, et al. Myeloid cell leukemia-1 inversely correlates with glycogen synthase kinase-3beta activity and associates with poor prognosis in human breast cancer. Cancer Res 2007; 67: 4564-4571.

28 Zheng H, Li W, Wang Y, Liu Z, Cai Y, Xie T, et al. Glycogen synthase kinase-3 beta regulates Snail and $\beta$-catenin expression during Fas-induced epithelialmesenchymal transition in gastrointestinal cancer. Eur $\mathbf{J}$ Cancer 2013; 49: 2734-2746.

29 McManus MT. MicroRNAs and cancer. Semin Cancer Biol 2003; 13: 253-258.

30 Pan X, Wang R, Wang ZX. The potential role of miR-451 in cancer diagnosis, prognosis, and therapy. Mol Cancer Ther 2013; 12: 1153-1162.

31 Liu X, Zhang X, Xiang J, Lv Y, Shi J. miR-451: potential role as tumor suppressor of human hepatoma cell growth and invasion. Int J Oncol 2014; 45: 739-745.

32 Li HY, Zhang Y, Cai JH, Bian HL. MicroRNA-451 inhibits growth of human colorectal carcinoma cells via downregulation of Pi3k/Akt pathway. Asian Pac J Cancer Prev 2013; 14: 3631-3634.

33 Tian Y, Nan Y, Han L, Zhang A, Wang G, Jia Z, et al. MicroRNA miR-451 downregulates the PI3K/AKT pathway through CAB39 in human glioma. Int J Oncol 2012; 40: 1105-1112.

34 Liu D, Liu C, Wang $\mathrm{X}$, Ingvarsson $\mathrm{S}$, Chen $\mathrm{H}$. MicroRNA-451 suppresses tumor cell growth by downregulating IL6R gene expression. Cancer Epidemiol 2014; 38: 85-92.

35 Godlewski J, Nowicki MO, Bronisz A, Nuovo G, Palatini J, De Lay M, et al. MicroRNA-451 regulates LKB1/AMPK signaling and allows adaptation to metabolic stress in glioma cells. Mol Cell 2010; 37: 620-632.

36 Godlewski J, Bronisz A, Nowicki MO, Chiocca EA, Lawler S. microRNA-451: A conditional switch controlling glioma cell proliferation and migration. Cell Cycle 2010; 9: 27422748.

37 Bian HB, Pan X, Yang JS, Wang ZX, De W. Upregulation of microRNA-451 increases cisplatin sensitivity of nonsmall cell lung cancer cell line (A549). J Exp Clin Cancer Res 2011; 30: 20.

38 Kovalchuk O, Filkowski J, Meservy J, Ilnytskyy Y, Tryndyak VP, Chekhun VF, et al. Involvement of microRNA-451 in resistance of the MCF-7 breast cancer cells to chemotherapeutic drug doxorubicin. Mol Cancer Ther 2008; 7: 2152-2159.

39 Bergamaschi A, Katzenellenbogen BS. Tamoxifen downregulation of miR-451 increases 14-3-3 $\zeta$ and promotes breast cancer cell survival and endocrine resistance. Oncogene 2012; 31: 39-47.

40 Lopotová T, Záčková M, Klamová H, Moravcová J. MicroRNA-451 in chronic myeloid leukemia: miR-451- 
BCR-ABL regulatory loop? Leuk Res 2011; 35: 974-977.

$41 \mathrm{Wu}$ Y, Zhou BP. New insights of epithelial-mesenchymal transition in cancer metastasis. Acta Biochim Biophys Sin (Shanghai) 2008; 40: 643-650.

42 Schliekelman MJ, Gibbons DL, Faca VM, Creighton CJ, Rizvi ZH, Zhang Q, et al. Targets of the tumor suppressor miR-200 in regulation of the epithelial-mesenchymal transition in cancer. Cancer Res 2011; 71: 7670-7682.

43 Kim NH, Kim HS, Li XY, Lee I, Choi HS, Kang SE, et al. A p53/miRNA-34 axis regulates Snail1-dependent cancer cell epithelial-mesenchymal transition. The Journal of cell biology 2011; 195: 417-433.

44 Zhang JP, Zeng C, Xu L, Gong J, Fang JH, Zhuang SM. MicroRNA-148a suppresses the epithelial-mesenchymal transition and metastasis of hepatoma cells by targeting Met/Snail signaling. Oncogene 2014; 33: 4069-4076.

45 Zhou Y, Li Y, Ye J, Jiang R, Yan H, Yang X, et al. MicroRNA-491 is involved in metastasis of hepatocellular carcinoma by inhibitions of matrix metalloproteinase and epithelial to mesenchymal transition. Liver Int 2013; 33: 1271-1280.

46 Yau WL, Lam CS, Ng L, Chow AK, Chan ST, Chan JY, et al. Over-expression of miR-106b promotes cell migration and metastasis in hepatocellular carcinoma by activating epithelial-mesenchymal transition process. PLoS One 2013; 8: e57882.

47 Xia H, Ooi LL, Hui KM. MicroRNA-216a/217-induced epithelial-mesenchymal transition targets PTEN and SMAD7 to promote drug resistance and recurrence of liver cancer. Hepatology 2013; 58: 629-641.

48 Peter ME. Targeting of mRNAs by multiple miRNAs: the next step. Oncogene 2010; 29: 2161-2164.

49 Dai MS, Lu H. Crosstalk between c-Myc and ribosome in ribosomal biogenesis and cancer. J Cell Biochem 2008; 105: 670-677.

50 Lin CP, Liu CR, Lee CN, Chan TS, Liu HE. Targeting c-Myc as a novel approach for hepatocellular carcinoma. World J Hepatol 2010; 2: 16-20.

51 Li X, Sanda T, Look AT, Novina CD, von Boehmer H. Repression of tumor suppressor miR-451 is essential for NOTCH1-induced oncogenesis in T-ALL. J Exp Med 2011; 208: 663-675.

52 Dang CV. MYC on the path to cancer. Cell 2012; 149: 2235.

53 Fenger JM, Bear MD, Volinia S, Lin TY, Harrington BK, London CA, et al. Overexpression of miR-9 in mast cells is associated with invasive behavior and spontaneous metastasis. BMC Cancer 2014; 14: 84.

54 Morimoto AM1, Tan N, West K, McArthur G, Toner GC, Manning WC, Smolich BD, et al. Gene expression profiling of human colon xenograft tumors following treatment with SU11248, a multitargeted tyrosine kinase inhibitor. Oncogene 2004; 23: 1618-1626.

55 Cui SY, Huang JY, Chen YT, Song HZ, Feng B, Huang
$\mathrm{GC}$, et al. Let-7c governs the acquisition of chemo- or radioresistance and epithelial-to-mesenchymal transition phenotypes in docetaxel-resistant lung adenocarcinoma. Mol Cancer Res 2013; 11: 699-713. 